hep-th/0601177

January, 2006

\title{
On compactified harmonic/projective superspace, 5D superconformal theories, and all that
}

\author{
Sergei M. Kuzenko ${ }^{1}$ \\ School of Physics M013, The University of Western Australia \\ 35 Stirling Highway, Crawley W.A. 6009, Australia
}

\begin{abstract}
Within the supertwistor approach, we analyse the superconformal structure of $4 \mathrm{D}$ $\mathcal{N}=2$ compactified harmonic/projective superspace. In the case of $5 \mathrm{D}$ superconformal symmetry, we derive the superconformal Killing vectors and related building blocks which emerge in the transformation laws of primary superfields. Various off-shell superconformal multiplets are presented both in 5D harmonic and projective superspaces, including the so-called tropical (vector) multiplet and polar (hyper)multiplet. Families of superconformal actions are described both in the 5D harmonic and projective superspace settings. We also present examples of 5D superconformal theories with gauged central charge.
\end{abstract}

\footnotetext{
${ }^{1}$ kuzenko@cyllene.uwa.edu.au
} 


\section{Introduction}

According to Nahm's classification [1], superconformal algebras exist in space-time dimensions $\mathrm{D} \leq 6$. Among the dimensions included, the case of $\mathrm{D}=5$ is truly exceptional, for it allows the existence of the unique superconformal algebra $F(4)[2]$. This is in drastic contrast to the other dimensions which are known to be compatible with series of superconformal algebras (say, 4D $\mathcal{N}$-extended or $6 \mathrm{D}(\mathcal{N}, 0)$ superconformal symmetry). Even on formal grounds, the exceptional feature of the five-dimensional case is interesting enough for studying in some depth the properties of 5D superconformal theories. On the other hand, such rigid superconformal theories are important prerequisites in the construction, within the superconformal tensor calculus [3, 4], of 5D supergravity-matter dynamical systems which are of primary importance, for example, in the context of bulkplus-brane scenarios.

The main motivation for the present work was the desire to develop a systematic setting to build 5D superconformal theories, and clearly this is hardly possible without employing superspace techniques. The superconformal algebra in five dimensions includes 5D simple (or $\mathcal{N}=1$ ) supersymmetry algebra ${ }^{1}$ as its super Poincaré subalgebra. As is well-known, for supersymmetric theories in various dimensions with eight supercharges (including the three important cases: (i) $4 \mathrm{D} \mathcal{N}=2$, (ii) $5 \mathrm{D} \mathcal{N}=1$ and (iii) $6 \mathrm{D} \mathcal{N}=(1,0)$ ) a powerful formalism to generate off-shell formulations is the harmonic superspace approach that was originally developed for the $4 \mathrm{D} \mathcal{N}=2$ supersymmertic Yang-Mills theories and supergravity $[5,6]$. There also exists a somewhat different, but related, formalism the so-called projective superspace approach [7, 8, 9, 10], first introduced soon after the harmonic superspace had appeared. Developed originally for describing the general selfcouplings of $4 \mathrm{D} \mathcal{N}=2$ tensor multiplets, this approach has been extended to include some other interesting multiplets. Both the harmonic and projective approaches make use of the same superspace, $\mathbb{R}^{D \mid 8} \times S^{2}$, which first emerged, for $D=4$, in a work of Rosly [11] (see also [12]) who built on earlier ideas due to Witten [13]. In harmonic superspace, one deals with so-called Grassmann analytic superfields required to be smooth tensor fields on $S^{2}$. In projective superspace, one also deals with Grassmann analytic superfields required, however, to be holomorphic on an open subset of $S^{2}$ (typically, the latter is chosen to be $\mathbb{C}^{*}=\mathbb{C} \backslash\{0\}$ in the Riemann sphere realisation $\left.S^{2}=\mathbb{C} \cup\{\infty\}\right)$. In many respects, the harmonic and projective superspaces are equivalent and complementary to each other [14], although harmonic superspace is obviously more fundamental. Keeping in

\footnotetext{
${ }^{1}$ On historic grounds, 5D simple supersymmetry is often labeled $\mathcal{N}=2$, see e.g. [4].
} 
mind potential applications to the brane-world physics, the projective superspace setting seems to be more useful, since the 5D projective supermultiplets [15] are easy to reduce to $4 \mathrm{D} \mathcal{N}=1$ superfields.

To our knowledge, no comprehensive discussion of the superconformal group and superconformal multiplets in projective superspace has been given, apart from the analysis of $S U(2)$ invariance in [7] and the semi-component consideration of tensor multiplets in [16]. On the contrary, a realisation of the superconformal symmetry in $4 \mathrm{D} \mathcal{N}=2$ harmonic superspace ${ }^{2}$ has been known for almost twenty years $[17,6]$. But some nuances of this realisation still appear to be quite mysterious (at least to newcomers) and call for a different interpretation. Specifically, one deals with superfields depending on harmonic variables $u_{i}^{ \pm}$subject to the two constraints

$$
u^{+i} u_{i}^{-}=1, \quad \overline{u^{+i}}=u_{i}^{-}, \quad i=\underline{1}, \underline{2}
$$

when describing general 4D $\mathcal{N}=2$ super Poincaré invariant theories in harmonic superspace $[5,6]$. In the case of superconformal theories, on the other hand, only the first constraint in (1.1) has to be imposed [17, 6]. Since any superconformal theory is, at the same time, a super Poincaré invariant one, some consistency issues seem to arise, such as that of the functional spaces used to describe the theory. It is quite remarkable that these issues simply do not occur if one pursues the twistor approach to harmonic superspace [19, 20, 21] (inspired by earlier constructions due to Manin [22]). In such a setting, the constraints (1.1) can be shown to appear only as possible 'gauge conditions' and therefore they have no intrinsic significance, with the only structural condition being $u^{+i} u_{i}^{-} \neq 0$. In our opinion, the supertwistor construction sketched in $[19,20]$ and further analysed in [21] is quite illuminating, for it allows a unified treatment of the harmonic and projective superspace formalisms. That is why it is reviewed and developed further in the present paper. Unlike [19, 21] and standard texts on Penrose's twistor theory [23], see e.g. [24], we avoid considering compactified complexified Minkowski space and its super-extensions, for these concepts are not relevant from the point of view of superconformal model-building we are interested in. Our 4D consideration is directly based on the use of (conformally) compactified Minkowski space $S^{1} \times S^{3}$ and its super-extensions. Compactified Minkowski space is quite interesting in its own right (see, e.g. [25]), and its universal covering space (i) possesses a unique causal structure compatible with conformal symmetry [26], and (ii) coincides with the boundary of five-dimensional Anti-de Sitter space, which is crucial in the context of the AdS/CFT duality [27].

\footnotetext{
${ }^{2}$ See $[18]$ for an extension to six dimensions.
} 
In the case of 5D superconformal symmetry, one can also pursue a supertwistor approach. However, since we are aiming at future applications to brane-world physics, a more pragmatic course is chosen here, which is based on the introduction of the relevant superconformal Killing vectors and elaborating associated building blocks. The concept of superconformal Killing vectors [28, 29, 30, 31, 32, 21, 33], has proved to be extremely useful for various studies of superconformal theories in four and six dimensions, see e.g. $[34,35,36]$.

This paper is organized as follows. In section 2 we review the construction [37] of compactified Minkowski space $\overline{\mathcal{M}}^{4}=S^{1} \times S^{3}$ as the set of null two-planes in the twistor space. In section 3 we discuss $\mathcal{N}$-extended compactified Minkowski superspace $\overline{\mathcal{M}}^{4 \mid 4 \mathcal{N}}$ and introduce the corresponding superconformal Killing vectors. In section 4 we develop different aspects of $4 \mathrm{D} \mathcal{N}=2$ compactified harmonic/projective superspace. Section 5 is devoted to the $5 \mathrm{D}$ superconformal formalism. Here we introduce the $5 \mathrm{D}$ superconformal Killing vectors and related building blocks, and also introduce several off-shell superconformal multiplets, both in the harmonic and projective superspaces. Section 6 introduces the zoo of 5D superconformal theories. Three technical appendices are also included at the end of the paper. In appendix A, a non-standard realisation for $S^{2}$ is given. Appendix $\mathrm{B}$ is devoted to the projective superspace action according to [8]. Some aspects of the reduction [15] from $5 \mathrm{D}$ projective supermultiplets to $4 \mathrm{D} \mathcal{N}=1,2$ superfields are collected in Appendix C.

\section{Compactified Minkowski space}

We start by recalling a remarkable realisation ${ }^{3}$ of compactified Minkowski space $\overline{\mathcal{M}}^{4}=$ $S^{1} \times S^{3}$ as the set of null two-dimensional subspaces in the twistor space ${ }^{4}$ which is a copy of $\mathbb{C}^{4}$. The twistor space is defined to be equipped with the scalar product

$$
\langle T, S\rangle=T^{\dagger} \Omega S, \quad \Omega=\left(\begin{array}{cc}
\mathbf{1}_{2} & 0 \\
0 & -\mathbf{1}_{2}
\end{array}\right),
$$

\footnotetext{
${ }^{3}$ This realisation is known in the physics literature since the early 1960's [37, 26, 38, 25], and it can be related (see, e.g. [26]) to the Weyl-Dirac construction [39, 40] of compactified Minkowski space $S^{1} \times S^{3} / \mathbb{Z}_{2}$ as the set of straight lines through the origin of the cone in $\mathbb{R}^{4,2}$. In the mathematics literature, its roots go back to Cartan's classification of the irreducible homogeneous bounded symmetric domains [41, 42].

${ }^{4}$ In the literature, the term 'twistor space' is often used for $\mathbb{C} P^{3}$. In this paper we stick to the original Penrose terminonology [23].
} 
for any twistors $T, S \in \mathbb{C}^{4}$. By construction, this scalar product is invariant under the action of the group $S U(2,2)$ to be identified with the conformal group. The elements of $S U(2,2)$ will be represented by block matrices

$$
g=\left(\begin{array}{ll}
A & B \\
C & D
\end{array}\right) \in S L(4, \mathbb{C}), \quad g^{\dagger} \Omega g=\Omega,
$$

where $A, B, C$ and $D$ are $2 \times 2$ matrices.

We will denote by $\overline{\mathcal{M}}^{4}$ the space of null two-planes through the origin in $\mathbb{C}^{4}$. Given a two-plane, it is generated by two linearly independent twistors $T^{\mu}$, with $\mu=1,2$, such that

$$
\left\langle T^{\mu}, T^{\nu}\right\rangle=0, \quad \mu, \nu=1,2 .
$$

Obviously, the basis chosen, $\left\{T^{\mu}\right\}$, is defined only modulo the equivalence relation

$$
\left\{T^{\mu}\right\} \sim\left\{\tilde{T}^{\mu}\right\}, \quad \tilde{T}^{\mu}=T^{\nu} R_{\nu}{ }^{\mu}, \quad R \in G L(2, \mathbb{C})
$$

Equivalently, the space $\overline{\mathcal{M}}^{4}$ consists of $4 \times 2$ matrices of rank two,

$$
\left(T^{\mu}\right)=\left(\begin{array}{c}
F \\
G
\end{array}\right), \quad F^{\dagger} F=G^{\dagger} G
$$

where $F$ and $G$ are $2 \times 2$ matrices defined modulo the equivalence relation

$$
\left(\begin{array}{l}
F \\
G
\end{array}\right) \sim\left(\begin{array}{l}
F R \\
G R
\end{array}\right), \quad R \in G L(2, \mathbb{C}) .
$$

In order for the two twistors $T^{\mu}$ in (2.5) to generate a two-plane, the $2 \times 2$ matrices $F$ and $G$ must be non-singular,

$$
\operatorname{det} F \neq 0, \quad \operatorname{det} G \neq 0 \text {. }
$$

Indeed, let us suppose the opposite. Then, the non-negative Hermitian matrix $F^{\dagger} F$ has a zero eigenvalue. Applying an equivalence transformation of the form

$$
\left(\begin{array}{l}
F \\
G
\end{array}\right) \rightarrow\left(\begin{array}{c}
F \mathcal{V} \\
G \mathcal{V}
\end{array}\right), \quad \mathcal{V} \in U(2)
$$

and therefore

$$
F^{\dagger} F \rightarrow \mathcal{V}^{-1}\left(F^{\dagger} F\right) \mathcal{V}, \quad G^{\dagger} G \rightarrow \mathcal{V}^{-1}\left(G^{\dagger} G\right) \mathcal{V}
$$


we can arrive at the following situation

$$
F^{\dagger} F=G^{\dagger} G=\left(\begin{array}{cc}
0 & 0 \\
0 & \lambda^{2}
\end{array}\right), \quad \lambda \in \mathbb{R} .
$$

In terms of the twistors $T^{\mu}$, the conditions obtained imply that $T^{1}=0$ and $T^{2} \neq 0$. But this contradicts the assumption that the two vectors $T^{\mu}$ generate a two-plane.

Because of (2.7), we have

$$
\left(\begin{array}{l}
F \\
G
\end{array}\right) \sim\left(\begin{array}{l}
h \\
\mathbf{1}
\end{array}\right), \quad h=F G^{-1} \in U(2) .
$$

It is seen that the space $\overline{\mathcal{M}}^{4}$ can be identified with the group manifold $U(2)=S^{1} \times S^{3}$.

The conformal group acts by linear transformations on the twistor space: associated with the group element (2.2) is the transformation $T \rightarrow g T$, for any twistor $T \in \mathbb{C}^{4}$. This group representation induces an action of $S U(2,2)$ on $\overline{\mathcal{M}}^{4}$. It is defined as follows:

$$
h \rightarrow g \cdot h=(A h+B)(C h+D)^{-1} \in U(2) .
$$

One can readily see that $\overline{\mathcal{M}}^{4}$ is a homogeneous space of the group $S U(2,2)$, and therefore it can be represented as $\overline{\mathcal{M}}^{4}=S U(2,2) / H_{h_{0}}$, where $H_{h_{0}}$ is the isotropy group at a fixed unitary matrix $h_{0} \in \overline{\mathcal{M}}^{4}$. With the choice

$$
h_{0}=-1
$$

a coset representative $s(h) \in S U(2,2)$ that maps $h_{0}$ to $h \in \overline{\mathcal{M}}^{4}$ can be chosen as follows (see, e.g. [43]):

$$
s(h)=(\operatorname{det} h)^{-1 / 4}\left(\begin{array}{cc}
-h & 0 \\
0 & \mathbf{1}
\end{array}\right), \quad s(h) \cdot h_{0}=h \in U(2) .
$$

The isotropy group corresponding to $h_{0}$ consists of those $S U(2,2)$ group elements $(2.2)$ which obey the requirement

$$
A+C=B+D
$$

This subgroup proves to be isomorphic to a group generated by the Lorentz transformations, dilatations and special conformal transformations. To visualise this, it is useful to implement a special similarity transformation for both the group $S U(2,2)$ and the twistor space. 
We introduce a special $4 \times 4$ matrix $\Sigma$,

$$
\Sigma=\frac{1}{\sqrt{2}}\left(\begin{array}{rr}
\mathbf{1}_{2} & -\mathbf{1}_{2} \\
\mathbf{1}_{2} & \mathbf{1}_{2}
\end{array}\right), \quad \Sigma^{\dagger} \Sigma=\mathbf{1}_{4},
$$

and associate with it the following similarity transformation:

$$
g \rightarrow \boldsymbol{g}=\Sigma g \Sigma^{-1}, \quad g \in S U(2,2) ; \quad T \rightarrow \boldsymbol{T}=\Sigma T, \quad T \in \mathbb{C}^{4}
$$

The elements of $S U(2,2)$ are now represented by block matrices

$$
\boldsymbol{g}=\left(\begin{array}{ll}
\boldsymbol{A} & \boldsymbol{B} \\
\boldsymbol{C} & \boldsymbol{D}
\end{array}\right) \in S L(4, \mathbb{C}), \quad \boldsymbol{g}^{\dagger} \boldsymbol{\Omega} \boldsymbol{g}=\boldsymbol{\Omega}
$$

where

$$
\Omega=\Sigma \Omega \Sigma^{-1}=\left(\begin{array}{cc}
0 & \mathbf{1}_{2} \\
\mathbf{1}_{2} & 0
\end{array}\right)
$$

The $2 \times 2$ matrices in (2.15) are related to those in (2.2) as follows:

$$
\begin{aligned}
\boldsymbol{A} & =\frac{1}{2}(A+D-B-C), \\
\boldsymbol{B} & =\frac{1}{2}(A+B-C-D), \\
\boldsymbol{C} & =\frac{1}{2}(A+C-B-D), \\
\boldsymbol{D} & =\frac{1}{2}(A+B+C+D) .
\end{aligned}
$$

Now, by comparing these expressions with (2.12) it is seen that the stability group $\Sigma H_{h_{0}} \Sigma^{-1}$ consists of upper block-triangular matrices,

$$
C=0
$$

When applied to $\overline{\mathcal{M}}^{4}$, the effect of the similarity transformation ${ }^{5}$ is

$$
\left(\begin{array}{l}
h \\
\mathbf{1}
\end{array}\right) \rightarrow \Sigma\left(\begin{array}{l}
h \\
\mathbf{1}
\end{array}\right)=\frac{1}{\sqrt{2}}\left(\begin{array}{c}
h-\mathbf{1} \\
h+\mathbf{1}
\end{array}\right) \sim\left(\begin{array}{c}
\mathbf{1} \\
-\mathrm{i} \tilde{x}
\end{array}\right), \quad \tilde{x}=x^{m}\left(\tilde{\sigma}_{m}\right)^{\dot{\alpha} \alpha}
$$

where

$$
-\mathrm{i} \tilde{x}=\frac{h+1}{h-1}, \quad \tilde{x}^{\dagger}=\tilde{x}
$$

\footnotetext{
${ }^{5}$ We follow the two-component spinor notation of Wess and Bagger [44].
} 
The inverse expression for $h$ in terms of $\tilde{x}$ is given by the so-called Cayley transform:

$$
-h=\frac{1-\mathrm{i} \tilde{x}}{1+\mathrm{i} \tilde{x}} .
$$

It is seen that

$$
h_{0}=-1 \quad \longleftrightarrow \quad \tilde{x}_{0}=0
$$

Unlike the original twistor representation, eqs. (2.5) and (2.7), the $2 \times 2$ matrices $h \pm \mathbf{1}$ in (2.19) may be singular at some points. This means that the variables $x^{m}(2.20)$ are welldefined local coordinates in the open subset of $\overline{\mathcal{M}}^{4}$ which is specified by $\operatorname{det}(h-\mathbf{1}) \neq 0$ and, as will become clear soon, can be identified with the ordinary Minkowski space.

As follows from (2.19), in terms of the variables $x^{m}$ the conformal group acts by fractional linear transformations

$$
-\mathrm{i} \tilde{x} \rightarrow-\mathrm{i} \tilde{x}^{\prime}=(\boldsymbol{C}-\mathrm{i} \boldsymbol{D} \tilde{x})(\boldsymbol{A}-\mathrm{i} \boldsymbol{B} \tilde{x})^{-1} .
$$

These transformations can be brought to a more familiar form if one takes into account the explicit structure of the elements of $S U(2,2)$ :

$$
\boldsymbol{g}=\mathrm{e}^{\boldsymbol{L}}, \quad \boldsymbol{L}=\left(\begin{array}{cc}
\omega_{\alpha}{ }^{\beta}-\frac{1}{2} \tau \delta_{\alpha}^{\beta} & -\mathrm{i} b_{\alpha \dot{\beta}} \\
-\mathrm{i} a^{\dot{\alpha} \beta} & -\bar{\omega}_{\dot{\beta}}^{\dot{\alpha}}+\frac{1}{2} \tau \delta_{\dot{\beta}}^{\dot{\alpha}}
\end{array}\right), \quad \boldsymbol{L}^{\dagger}=-\boldsymbol{\Omega} \boldsymbol{L} \boldsymbol{\Omega}
$$

Here the matrix elements correspond to a Lorentz transformation $\left(\omega_{\alpha}^{\beta}, \bar{\omega}^{\dot{\alpha}}\right)$, translation

$a^{\dot{\alpha} \beta}$, special conformal transformation $b_{\alpha \dot{\beta}}$ and dilatation $\tau$. In accordance with (2.18), the isotropy group at $x_{0}=0$ is spanned by the Lorentz transformations, special conformal boosts and scale transformations.

\section{Compactified Minkowski superspace}

The construction reviewed in the previous section can be immediately generalised to the case of $\mathcal{N}$-extended conformal supersymmetry [22], by making use of the supertwistor space $\mathbb{C}^{4 \mid \mathcal{N}}$ introduced by Ferber [45], with $\mathcal{N}=1,2,3$ (the case $\mathcal{N}=4$ is known to be somewhat special, and will not be discussed here). The supertwistor space is equipped with scalar product

$$
\langle T, S\rangle=T^{\dagger} \Omega S, \quad \Omega=\left(\begin{array}{ccc}
\mathbf{1}_{2} & 0 \\
& -\mathbf{1}_{2} & \\
0 & & -\mathbf{1}_{\mathcal{N}}
\end{array}\right)
$$


for any supertwistors $T, S \in \mathbb{C}^{4 \mid \mathcal{N}}$. The $\mathcal{N}$-extended superconformal group acting on the supertwistor space is $S U(2,2 \mid \mathcal{N})$. It is spanned by supermatrices of the form

$$
g \in S L(4 \mid \mathcal{N}), \quad g^{\dagger} \Omega g=\Omega .
$$

In complete analogy with the bosonic construction, compactified Minkowski superspace $\overline{\mathcal{M}}^{4 \mid 4 \mathcal{N}}$ is defined to be the space of null two-planes through the origin in $\mathbb{C}^{4 \mid \mathcal{N}}$. Given such a two-plane, it is generated by two supertwistors $T^{\mu}$ such that (i) their bodies are linearly independent; (ii) they obey the equations (2.3) and (2.4). Equivalently, the space $\overline{\mathcal{M}}^{4 \mid 4 \mathcal{N}}$ consists of rank-two supermatrices of the form

$$
\left(T^{\mu}\right)=\left(\begin{array}{c}
F \\
G \\
\Upsilon
\end{array}\right), \quad F^{\dagger} F=G^{\dagger} G+\Upsilon^{\dagger} \Upsilon
$$

defined modulo the equivalence relation

$$
\left(\begin{array}{l}
F \\
G \\
\Upsilon
\end{array}\right) \sim\left(\begin{array}{l}
F R \\
G R \\
\Upsilon R
\end{array}\right), \quad R \in G L(2, \mathbb{C})
$$

Here $F$ and $G$ are $2 \times 2$ bosonic matrices, and $\Upsilon$ is a $\mathcal{N} \times 2$ fermionic matrix. As in the bosonic case, we have

$$
\left(\begin{array}{l}
F \\
G \\
\Upsilon
\end{array}\right) \sim\left(\begin{array}{c}
h \\
1 \\
\Theta
\end{array}\right), \quad h^{\dagger} h=\mathbf{1}+\Theta^{\dagger} \Theta
$$

Introduce the supermatrix

$$
\Sigma=\frac{1}{\sqrt{2}}\left(\begin{array}{ccc}
\mathbf{1}_{2} & -\mathbf{1}_{2} & 0 \\
\mathbf{1}_{2} & \mathbf{1}_{2} & 0 \\
0 & 0 & \sqrt{2} \mathbf{1}_{\mathcal{N}}
\end{array}\right), \quad \Sigma^{\dagger} \Sigma=\mathbf{1}_{4+\mathcal{N}}
$$

and associate with it the following similarity transformation:

$$
g \rightarrow \boldsymbol{g}=\Sigma g \Sigma^{-1}, \quad g \in S U(2,2 \mid \mathcal{N}) ; \quad T \rightarrow \boldsymbol{T}=\Sigma T, \quad T \in \mathbb{C}^{4 \mid \mathcal{N}} .
$$

The supertwistor metric becomes

$$
\boldsymbol{\Omega}=\Sigma \Omega \Sigma^{-1}=\left(\begin{array}{ccc}
0 & \mathbf{1}_{2} & 0 \\
\mathbf{1}_{2} & 0 & 0 \\
0 & 0 & -\mathbf{1}_{\mathcal{N}}
\end{array}\right) .
$$


When implemented on the superspace $\overline{\mathcal{M}}^{4 \mid 4 \mathcal{N}}$, the similarity transformation results in

$$
\left(\begin{array}{c}
h \\
\mathbf{1} \\
\Theta
\end{array}\right) \rightarrow \Sigma\left(\begin{array}{c}
h \\
\mathbf{1} \\
\Theta
\end{array}\right)=\frac{1}{\sqrt{2}}\left(\begin{array}{c}
h-\mathbf{1} \\
h+\mathbf{1} \\
\sqrt{2} \Theta
\end{array}\right) \sim\left(\begin{array}{c}
\mathbf{1} \\
-\mathrm{i} \tilde{x}_{+} \\
2 \theta
\end{array}\right)=\left(\begin{array}{c}
\delta_{\alpha}^{\beta} \\
-\mathrm{i} \tilde{x}_{+}^{\dot{\alpha} \beta} \\
2 \theta_{i}^{\beta}
\end{array}\right)
$$

where

$$
-\mathrm{i} \tilde{x}_{+}=\frac{h+\mathbf{1}}{h-\mathbf{1}}, \quad \sqrt{2} \theta=\Theta(h-\mathbf{1})^{-1} .
$$

The bosonic $\tilde{x}_{+}$and fermionic $\theta$ variables obey the reality condition

$$
\tilde{x}_{+}-\tilde{x}_{-}=4 \mathrm{i} \theta^{\dagger} \theta, \quad \tilde{x}_{-}=\left(\tilde{x}_{+}\right)^{\dagger}
$$

It is solved by

$$
x_{ \pm}^{\dot{\alpha} \beta}=x^{\dot{\alpha} \beta} \pm 2 \mathrm{i} \bar{\theta}^{\dot{\alpha} i} \theta_{i}^{\beta}, \quad \bar{\theta}^{\dot{\alpha} i}=\overline{\theta_{i}^{\alpha}}, \quad \tilde{x}^{\dagger}=\tilde{x}
$$

with $z^{A}=\left(x^{a}, \theta_{i}^{\alpha}, \bar{\theta}_{\dot{\alpha}}^{i}\right)$ the coordinates of $\mathcal{N}$-extended flat global superspace $\mathbb{R}^{4 \mid 4 \mathcal{N}}$. We therefore see that the supertwistors in (3.9) are parametrized by the variables $x_{+}^{a}$ and $\theta_{i}^{\alpha}$ which are the coordinates in the chiral subspace. Since the superconformal group acts by linear transformations on $\mathbb{C}^{4 \mid 2 \mathcal{N}}$, we can immediately conclude that it acts by holomorphic transformations on the chiral subspace.

To describe the action of $S U(2,2 \mid \mathcal{N})$ on the chiral subspace, let us consider a generic group element:

$$
\boldsymbol{g}=\mathrm{e}^{\boldsymbol{L}}, \quad \boldsymbol{L}=\left(\begin{array}{ccc}
\omega_{\alpha}{ }^{\beta}-\sigma \delta_{\alpha}{ }^{\beta} & -\mathrm{i} b_{\alpha \dot{\beta}} & 2 \eta_{\alpha}{ }^{j} \\
-\mathrm{i} a^{\dot{\alpha} \beta} & -\bar{\omega}^{\dot{\alpha}}{ }_{\dot{\beta}}+\bar{\sigma} \delta^{\dot{\alpha}} \dot{\beta} & 2 \bar{\epsilon}^{\dot{\alpha} j} \\
2 \epsilon_{i}{ }^{\beta} & 2 \bar{\eta}_{i \dot{\beta}} & \frac{2}{\mathcal{N}}(\bar{\sigma}-\sigma) \delta_{i}{ }^{j}+\Lambda_{i}^{j}
\end{array}\right)
$$

where

$$
\sigma=\frac{1}{2}\left(\tau+\mathrm{i} \frac{\mathcal{N}}{\mathcal{N}-4} \varphi\right), \quad \Lambda^{\dagger}=-\Lambda, \quad \operatorname{tr} \Lambda=0
$$

Here the matrix elements, which are not present in (2.24), correspond to a $Q$-supersymmetry $\left(\epsilon_{i}^{\alpha}, \bar{\epsilon}^{\dot{\alpha} i}\right), S$-supersymmetry $\left(\eta_{\alpha}^{i}, \bar{\eta}_{i \dot{\alpha}}\right)$, combined scale and chiral transformation $\sigma$, and chiral $S U(\mathcal{N})$ transformation $\Lambda_{i}{ }^{j}$. Now, one can check that the coordinates of the chiral subspace transform as follows:

$$
\begin{aligned}
\delta \tilde{x}_{+} & =\tilde{a}+(\sigma+\bar{\sigma}) \tilde{x}_{+}-\bar{\omega} \tilde{x}_{+}-\tilde{x}_{+} \omega+\tilde{x}_{+} b \tilde{x}_{+}+4 \mathrm{i} \bar{\epsilon} \theta-4 \tilde{x}_{+} \eta \theta \\
\delta \theta & =\epsilon+\frac{1}{\mathcal{N}}((\mathcal{N}-2) \sigma+2 \bar{\sigma}) \theta-\theta \omega+\Lambda \theta+\theta b \tilde{x}_{+}-\mathrm{i} \bar{\eta} \tilde{x}_{+}-4 \theta \eta \theta .
\end{aligned}
$$


Expressions (3.15) can be rewritten in a more compact form,

$$
\delta x_{+}^{a}=\xi_{+}^{a}\left(x_{+}, \theta\right), \quad \delta \theta_{i}^{\alpha}=\xi_{i}^{\alpha}\left(x_{+}, \theta\right)
$$

where

$$
\xi_{+}^{a}=\xi^{a}+\frac{\mathrm{i}}{8} \xi_{i} \sigma^{a} \bar{\theta}^{i}, \quad \overline{\xi^{a}}=\xi^{a} .
$$

Here the parameters $\xi^{a}$ and $\xi_{i}^{\alpha}$ are components of the superconformal Killing vector

$$
\xi=\bar{\xi}=\xi^{a}(z) \partial_{a}+\xi_{i}^{\alpha}(z) D_{\alpha}^{i}+\bar{\xi}_{\dot{\alpha}}^{i}(z) \bar{D}_{i}^{\dot{\alpha}}
$$

which generates the infinitesimal transformation in the full superspace, $z^{A} \rightarrow z^{A}+\xi z^{A}$, and is defined to satisfy

$$
\left[\xi, \bar{D}_{i}^{\dot{\alpha}}\right] \propto \bar{D}_{j}^{\dot{\beta}}
$$

and therefore

$$
\bar{D}_{i}^{\dot{\alpha}} \xi^{\dot{\beta} \beta}=4 \mathrm{i} \varepsilon^{\dot{\alpha} \dot{\beta}} \xi_{i}^{\beta}
$$

All information about the superconformal algebra is encoded in the superconformal Killing vectors. From eq. (3.20) it follows that

$$
\left[\xi, D_{\alpha}^{i}\right]=-\left(D_{\alpha}^{i} \xi_{j}^{\beta}\right) D_{\beta}^{j}=\tilde{\omega}_{\alpha}^{\beta} D_{\beta}^{i}-\frac{1}{\mathcal{N}}((\mathcal{N}-2) \tilde{\sigma}+2 \overline{\tilde{\sigma}}) D_{\alpha}^{i}-\tilde{\Lambda}_{j}^{i} D_{\alpha}^{j} .
$$

Here the parameters of 'local' Lorentz $\tilde{\omega}$ and scale-chiral $\tilde{\sigma}$ transformations are

$$
\tilde{\omega}_{\alpha \beta}(z)=-\frac{1}{\mathcal{N}} D_{(\alpha}^{i} \xi_{\beta) i}, \quad \tilde{\sigma}(z)=\frac{1}{\mathcal{N}(\mathcal{N}-4)}\left(\frac{1}{2}(\mathcal{N}-2) D_{\alpha}^{i} \xi_{i}^{\alpha}-\bar{D}_{i}^{\dot{\alpha}} \bar{\xi}_{\dot{\alpha}}^{i}\right)
$$

and turn out to be chiral

$$
\bar{D}_{\dot{\alpha} i} \tilde{\omega}_{\alpha \beta}=0, \quad \bar{D}_{\dot{\alpha} i} \tilde{\sigma}=0
$$

The parameters $\tilde{\Lambda}_{j}^{i}$

$$
\tilde{\Lambda}_{j}{ }^{i}(z)=-\frac{\mathrm{i}}{32}\left(\left[D_{\alpha}^{i}, \bar{D}_{\dot{\alpha} j}\right]-\frac{1}{\mathcal{N}} \delta_{j}{ }^{i}\left[D_{\alpha}^{k}, \bar{D}_{\dot{\alpha} k}\right]\right) \xi^{\dot{\alpha} \alpha}, \quad \tilde{\Lambda}^{\dagger}=-\tilde{\Lambda}, \quad \operatorname{tr} \tilde{\Lambda}=0
$$

correspond to 'local' $S U(\mathcal{N})$ transformations. One can readily check the identity

$$
D_{\alpha}^{k} \tilde{\Lambda}_{j}^{i}=-2\left(\delta_{j}^{k} D_{\alpha}^{i}-\frac{1}{\mathcal{N}} \delta_{j}^{i} D_{\alpha}^{k}\right) \tilde{\sigma}
$$




\section{Compactified harmonic/projective superspace}

For Ferber's supertwistors used in the previous section, a more appropriate name seems to be even supertwistors. Being elements of $\mathbb{C}^{4 \mid \mathcal{N}}$, these objects have four bosonic components and $\mathcal{N}$ fermionic components. One can also consider odd supertwistors [20]. By definition, these are $4+\mathcal{N}$ vector-columns such that their top four entries are fermionic, and the rest $\mathcal{N}$ components are bosonic. In other words, the odd supertwistors are elements of $\mathbb{C}^{\mathcal{N} \mid 4}$. It is natural to treat the even and odd supertwistors as the even and odd elements, respectively, of a supervector space ${ }^{6}$ of dimension $(4 \mid \mathcal{N})$ on which the superconformal group $S U(2,2 \mid \mathcal{N})$ acts. Both even and odd supertwistors should be used $[19,20]$ in order to define harmonic-like superspaces in extended supersymmetry.

Throughout this section, our consideration is restricted to the case $\mathcal{N}=2$. Then, $\tilde{\Lambda}^{i j}=\varepsilon^{i k} \tilde{\Lambda}_{k}^{j}$ is symmetric, $\tilde{\Lambda}^{i j}=\tilde{\Lambda}^{j i}$, and eq. (3.25) implies

$$
D_{\alpha}^{(i} \tilde{\Lambda}^{j k)}=\bar{D}_{\dot{\alpha}}^{(i} \tilde{\Lambda}^{j k)}=0
$$

\subsection{Projective realisation}

Following [20], we accompany the two even null supertwistors $T^{\mu}$, which occur in the construction of the compactified $\mathcal{N}=2$ superspace $\overline{\mathcal{M}}^{4 \mid 8}$, by an odd supertwistor $\Xi$ with non-vanishing body (in particular, the body of $\langle\Xi, \Xi\rangle$ is non-zero). These supertwistors are required to obey

$$
\left\langle T^{\mu}, T^{\nu}\right\rangle=\left\langle T^{\mu}, \Xi\right\rangle=0, \quad \mu, \nu=1,2,
$$

and are defined modulo the equivalence relation

$$
\left(\Xi, T^{\mu}\right) \sim\left(\Xi, T^{\nu}\right)\left(\begin{array}{cc}
c & 0 \\
\rho_{\nu} & R_{\nu}{ }^{\mu}
\end{array}\right), \quad\left(\begin{array}{cc}
c & 0 \\
\rho & R
\end{array}\right) \in G L(1 \mid 2),
$$

with $\rho_{\nu}$ anticommuting complex parameters. The superspace obtained can be seen to be $\overline{\mathcal{M}}^{4 \mid 8} \times S^{2}$. Indeed, using the above freedom in the definition of $T^{\mu}$ and $\Xi$, we can choose them to be of the form

$$
T^{\mu} \sim\left(\begin{array}{c}
h \\
1 \\
\Theta
\end{array}\right), \quad \Xi \sim\left(\begin{array}{c}
0 \\
-\Theta^{\dagger} v \\
v
\end{array}\right), \quad h^{\dagger} h=\mathbf{1}+\Theta^{\dagger} \Theta, \quad v \neq 0 .
$$

Here the non-zero two-vector $v \in \mathbb{C}^{2}$ is still defined modulo re-scalings $v \rightarrow c v$, with $c \in \mathbb{C}^{*}$. A natural name for the supermanifold obtained is projective superspace.

\footnotetext{
${ }^{6}$ See, e.g. $[46,32]$ for reviews on supervector spaces.
} 


\subsection{Harmonic realisation}

Now, we would like to present a somewhat different, but equivalent, realisation for $\overline{\mathcal{M}}^{4 \mid 8} \times S^{2}$ inspired by the exotic realisation for the two-sphere described in Appendix A. We will consider a space of quadruples $\left\{T^{\mu}, \Xi^{+}, \Xi^{-}\right\}$consisting of two even supertwistors $T^{\mu}$ and two odd supertwistors $\Xi^{ \pm}$such that (i) the bodies of $T^{\mu}$ are linearly independent four-vectors; (ii) the bodies of $\Xi^{ \pm}$are lineraly independent two-vectors. These supertwistors are further required to obey the relations

$$
\left\langle T^{\mu}, T^{\nu}\right\rangle=\left\langle T^{\mu}, \Xi^{+}\right\rangle=\left\langle T^{\mu}, \Xi^{-}\right\rangle=0, \quad \mu, \nu=1,2
$$

and are defined modulo the equivalence relation

$$
\left(\Xi^{-}, \Xi^{+}, T^{\mu}\right) \sim\left(\Xi^{-}, \Xi^{+}, T^{\nu}\right)\left(\begin{array}{ccc}
a & 0 & 0 \\
b & c & 0 \\
\rho_{\nu}^{-} & \rho_{\nu}^{+} & R_{\nu}{ }^{\mu}
\end{array}\right), \quad\left(\begin{array}{ccc}
a & 0 & 0 \\
b & c & 0 \\
\rho^{-} & \rho^{+} & R
\end{array}\right) \in G L(2 \mid 2),
$$

with $\rho_{\nu}^{ \pm}$anticommuting complex parameters. Using the 'gauge freedom' in the definition of $T^{\mu}$ and $\Xi^{ \pm}$, these supertwistors can be chosen to have the form

$$
T^{\mu} \sim\left(\begin{array}{c}
h \\
\mathbf{1} \\
\Theta
\end{array}\right), \quad \Xi^{ \pm} \sim\left(\begin{array}{c}
0 \\
-\Theta^{\dagger} v^{ \pm} \\
v^{ \pm}
\end{array}\right), \quad h^{\dagger} h=\mathbf{1}+\Theta^{\dagger} \Theta, \quad \operatorname{det}\left(v^{-} v^{+}\right) \neq 0
$$

Here the 'complex harmonics' $v^{ \pm}$are still defined modulo arbitrary transformations of the form (A.9). Given a $2 \times 2$ matrix $\boldsymbol{v}=\left(v^{-} v^{+}\right) \in G L(2, \mathbb{C})$, there always exists a lower triangular matrix $R$ such that $\boldsymbol{v} R \in S U(2)$. The latter implies that $v^{-}$is uniquely determined in terms of $v^{+}$, and therefore the supermanifold under consideration is indeed $\overline{\mathcal{M}}^{4 \mid 8} \times S^{2}$. In accordance with the construction given, a natural name for this supermanifold is harmonic superspace.

\subsection{Embedding of $\mathbb{R}^{4 \mid 8} \times S^{2}$ : Harmonic realisation}

We can now analyse the structure of superconformal transformations on the flat global superspace $\mathbb{R}^{4 \mid 8} \times S^{2}$ embedded in $\overline{\mathcal{M}}^{4 \mid 8} \times S^{2}$.

Upon implementing the similarity transformation, eq. (3.7), we have

$$
\left(\boldsymbol{T}^{\mu}\right) \sim\left(\begin{array}{c}
\mathbf{1} \\
-\mathrm{i} \tilde{x}_{+} \\
2 \theta
\end{array}\right)=\left(\begin{array}{c}
\delta_{\alpha}{ }^{\beta} \\
-\mathrm{i} \tilde{x}_{+}^{\dot{\alpha} \beta} \\
2 \theta_{i}{ }^{\beta}
\end{array}\right), \quad \boldsymbol{\Xi}^{ \pm} \sim\left(\begin{array}{c}
0 \\
2 \bar{\theta}^{ \pm} \\
u^{ \pm}
\end{array}\right)=\left(\begin{array}{c}
0 \\
2 \bar{\theta}^{ \pm \dot{\alpha}} \\
u_{i}^{ \pm}
\end{array}\right) .
$$


with

$$
\operatorname{det}\left(u_{i}{ }^{-} u_{i}{ }^{+}\right)=u^{+i} u_{i}^{-} \neq 0, \quad u^{+i}=\varepsilon^{i j} u_{j}^{+} .
$$

Here the bosonic $x_{+}^{m}$ and fermionic $\theta_{i}^{\alpha}$ variables are related to each other by the reality condition (3.11). The orthogonality conditions $\left\langle\boldsymbol{T}^{\mu}, \boldsymbol{\Xi}^{ \pm}\right\rangle=0$ imply

$$
\bar{\theta}^{+\dot{\alpha}}=\bar{\theta}^{\dot{\alpha} i} u_{i}^{+}, \quad \bar{\theta}^{-\dot{\alpha}}=\bar{\theta}^{\dot{\alpha} i} u_{i}^{-} .
$$

The complex harmonic variables $u_{i}^{ \pm}$in (4.8) are still defined modulo arbitrary transformations of the form

$$
\left(u_{i}{ }^{-} u_{i}{ }^{+}\right) \rightarrow\left(u_{i}{ }^{-} u_{i}{ }^{+}\right) R, \quad R=\left(\begin{array}{ll}
a & 0 \\
b & c
\end{array}\right) \in G L(2, \mathbb{C}) .
$$

The 'gauge' freedom (4.10) can be reduced by imposing the 'gauge' condition

$$
u^{+i} u_{i}^{-}=1 \text {. }
$$

It can be further reduced by choosing the harmonics to obey the reality condition

$$
u^{+i}=\overline{u_{i}^{-}} .
$$

Both requirements (4.11) and (4.12) have no fundamental significance, and represent themselves possible gauge conditions only. It is worth pointing out that the reality condition (4.12) implies $\left\langle\boldsymbol{\Xi}^{-}, \boldsymbol{\Xi}^{+}\right\rangle=0$. If both equations (4.11) and (4.12) hold, then we have in addition $\left\langle\boldsymbol{\Xi}^{+}, \boldsymbol{\Xi}^{+}\right\rangle=\left\langle\boldsymbol{\Xi}^{-}, \boldsymbol{\Xi}^{-}\right\rangle=-1$.

In what follows, the harmonics will be assumed to obey eq. (4.11) only. As explained in the appendix, the gauge freedom (4.10) allows one to represent any infinitesimal transformation of the harmonics as follows:

$$
\delta u_{i}^{-}=0, \quad \delta u_{i}^{+}=\rho^{++}(u) u_{i}^{-}
$$

for some parameter $\rho^{++}$which is determined by the transformation under consideration.

In the case of an infinitesimal superconformal transformation (3.13), one derives

$$
\delta u_{i}^{-}=0, \quad \delta u_{i}^{+}=-\tilde{\Lambda}^{++} u_{i}^{-}, \quad \tilde{\Lambda}^{++}=\tilde{\Lambda}^{i j} u_{i}^{+} u_{j}^{+}
$$

with the parameter $\tilde{\Lambda}^{i j}$ given by (3.24). Due to (4.1), we have (using the notation $D_{\alpha}^{ \pm}=$ $D_{\alpha}^{i} u_{i}^{ \pm}$and $\left.\bar{D}_{\dot{\alpha}}^{ \pm}=\bar{D}_{\dot{\alpha}}^{i} u_{i}^{ \pm}\right)$

$$
D_{\alpha}^{+} \tilde{\Lambda}^{++}=\bar{D}_{\dot{\alpha}}^{+} \tilde{\Lambda}^{++}=0, \quad D^{++} \tilde{\Lambda}^{++}=0 .
$$


Here and below, we make use of the harmonic derivatives [5]

$$
D^{++}=u^{+i} \frac{\partial}{\partial u^{-i}}, \quad D^{--}=u^{-i} \frac{\partial}{\partial u^{+i}} .
$$

It is not difficult to express $\tilde{\Lambda}^{++}$in terms of the parameters in (3.13) and superspace coordinates:

$$
\tilde{\Lambda}^{++}=\Lambda^{i j} u_{i}^{+} u_{j}^{+}+4 \mathrm{i} \theta^{+} b \bar{\theta}^{+}-\left(\theta^{+} \eta^{+}-\bar{\theta}^{+} \bar{\eta}^{+}\right) .
$$

The transformation (4.13) coincides with the one originally given in [17].

For the superconformal variations of $\theta_{\alpha}^{+}$and $\bar{\theta}_{\dot{\alpha}}^{+}$, one finds

$$
\delta \theta_{\alpha}^{+}=\delta \theta_{\alpha}^{i} u_{i}^{+}+\theta_{\alpha}^{i} \delta u_{i}^{+}=\xi_{\alpha}^{i} u_{i}^{+}-\tilde{\Lambda}^{++} \theta_{\alpha}^{i} u_{i}^{-},
$$

and similarly for $\delta \bar{\theta}_{\dot{\alpha}}^{+}$. From eqs. (3.21) and (4.14) one then deduces

$$
D_{\beta}^{+} \delta q_{\alpha}^{+}=\bar{D}_{\dot{\beta}}^{+} \delta \theta_{\alpha}^{+}=0
$$

and similarly for $\delta \bar{\theta}_{\dot{\alpha}}^{+}$. The superconformal variations $\delta q_{\alpha}^{+}$and $\delta \bar{\theta}_{\dot{\alpha}}^{+}$can be seen to coincide with those originally given in [17]. One can also check that the superconformal variation of the analytic bosonic coordinates

$$
y^{a}=x^{a}-2 \mathrm{i} \theta^{(i} \sigma^{a} \bar{\theta}^{j)} u_{i}^{+} u_{j}^{-}, \quad D_{\beta}^{+} y^{a}=\bar{D}_{\dot{\beta}}^{+} y^{a}=0,
$$

is analytic. This actually follows from the transformation

$$
\delta D_{\alpha}^{+} \equiv\left[\xi-\tilde{\Lambda}^{++} D^{--}, D_{\alpha}^{+}\right]=\tilde{\omega}_{\alpha}^{\beta} D_{\beta}^{+}-\left(\tilde{\sigma}+\tilde{\Lambda}^{i j} u_{i}^{+} u_{j}^{-}\right) D_{\alpha}^{+},
$$

and similarly for $\delta \bar{D}_{\dot{\alpha}}^{+}$.

We conclude that the analytic subspace parametrized by the variables

$$
\zeta=\left(y^{a}, \theta^{+\alpha}, \bar{\theta}_{\dot{\alpha}}^{+}, u_{i}^{+}, u_{j}^{-}\right), \quad D_{\beta}^{+} \zeta=\bar{D}_{\dot{\beta}}^{+} \zeta=0,
$$

is invariant under the superconformal group. The superconformal variations of these coordinates coincide with those given in [17]. No consistency clash occurs between the $S U(2)$-type constraints (1.1) and the superconformal transformation law (4.13), because the construction does not require imposing either of the constraints (1.1).

Using eq. (3.25) one can show that the following descendant of the superconformal Killing vector

$$
\Sigma=\tilde{\Lambda}^{i j} u_{i}^{+} u_{j}^{-}+\tilde{\sigma}+\overline{\tilde{\sigma}}
$$

possesses the properties

$$
D_{\beta}^{+} \Sigma=\bar{D}_{\dot{\beta}}^{+} \Sigma=0, \quad D^{++} \Sigma=\tilde{\Lambda}^{++} .
$$

It turns out that the objects $\xi, \tilde{\Lambda}^{++}$and $\Sigma$ determine the superconformal transformations of primary analytic superfields [6]. 


\subsection{Embedding of $\mathbb{R}^{4 \mid 8} \times S^{2}$ : Projective realisation}

Now, let us try to exploit the realisation of $S^{2}$ as the Riemann sphere $\mathbb{C} P^{1}$. The superspace can be covered by two open sets - the north chart and the south chart - that are specified by the conditions: (i) $u^{+1} \neq 0$; and (ii) $u^{+\underline{2}} \neq 0$.

In the north chart, the gauge freedom (4.10) can be completely fixed by choosing

$$
\begin{aligned}
& u^{+i} \sim(1, w) \equiv w^{i}, \quad u_{i}^{+} \sim(-w, 1)=w_{i}, \\
& u^{-i} \sim(0,-1), \quad u_{i}^{-} \sim(1,0) .
\end{aligned}
$$

Here $w$ is the complex coordinate parametrizing the north chart. Then the transformation law (4.13) turns into

$$
\delta w=\tilde{\Lambda}^{++}(w), \quad \tilde{\Lambda}^{++}(w)=\tilde{\Lambda}^{i j} w_{i}^{+} w_{j}^{+}
$$

It is seen that the superconformal group acts by holomorphic transformations.

The south chart is defined by

$$
\begin{aligned}
u^{+i} \sim(y, 1) \equiv y^{i}, & u_{i}^{+} \sim(-1, y)=y_{i} \\
u^{-i} \sim(1,0), & u_{i}^{-} \sim(0,1)
\end{aligned}
$$

with $y$ the local complex coordinate. The transformation law (4.13) becomes

$$
\delta y=-\tilde{\Lambda}^{++}(y), \quad \tilde{\Lambda}^{++}(y)=\tilde{\Lambda}^{i j} y_{i}^{+} y_{j}^{+}
$$

In the overlap of the north and south charts, the corresponding complex coordinates are related to each other in the standard way:

$$
y=\frac{1}{w} .
$$

\section{5D superconformal formalism}

As we have seen, modulo some global topological issues, all information about the superconformal structures in a superspace is encoded in the corresponding superconformal Killing vectors. In developing the 5D superconformal formalism below, we will not pursue global aspects, and simply base our consideration upon elaborating the superconformal Killing vectors and related concepts. Our 5D notation and conventions follow [15]. 


\subsection{D superconformal Killing vectors}

In $5 \mathrm{D}$ simple superspace $\mathbb{R}^{5 \mid 8}$ parametrized by coordinates $z^{\hat{A}}=\left(x^{\hat{a}}, \theta_{i}^{\hat{\alpha}}\right)$, we introduce an infinitesimal coordinate transformation

$$
z^{\hat{A}} \rightarrow z^{\hat{A}}=z^{\hat{A}}+\xi z^{\hat{A}}
$$

generated by a real vector field

$$
\xi=\bar{\xi}=\xi^{\hat{a}}(z) \partial_{\hat{a}}+\xi_{i}^{\hat{\alpha}}(z) D_{\hat{\alpha}}^{i}
$$

with $D_{\hat{A}}=\left(\partial_{\hat{a}}, D_{\hat{\alpha}}^{i}\right)$ the flat covariant derivatives. The transformation is said to be superconformal if $\left[\xi, D_{\hat{\alpha}}^{i}\right] \propto D_{\hat{\beta}}^{j}$, or more precisely

$$
\left[\xi, D_{\hat{\alpha}}^{i}\right]=-\left(D_{\hat{\alpha}}^{i} \xi_{j}^{\hat{\beta}}\right) D_{\hat{\beta}}^{j}
$$

The latter equation is equivalent to

$$
D_{\hat{\alpha}}^{i} \xi^{\hat{b}}=2 \mathrm{i}\left(\Gamma^{\hat{b}}\right)_{\hat{\alpha}}^{\hat{\beta}} \xi_{\hat{\beta}}^{i}=-2 \mathrm{i}\left(\Gamma^{\hat{b}}\right)_{\hat{\alpha} \hat{\beta}} \xi^{\hat{\beta} i}
$$

It follows from here

$$
\varepsilon^{i j}\left(\Gamma_{\hat{a}}\right)_{\hat{\alpha} \hat{\beta}} \partial^{\hat{a}} \xi^{\hat{b}}=\left(\Gamma^{\hat{b}}\right)_{\hat{\alpha} \hat{\gamma}} D_{\hat{\beta}}^{j} \xi^{\hat{\gamma} i}+\left(\Gamma^{\hat{b}}\right)_{\hat{\beta} \hat{\gamma}} D_{\hat{\alpha}}^{i} \xi^{\hat{\gamma} j}
$$

This equation implies that $\xi^{\hat{a}}=\xi^{\hat{a}}(x, \theta)$ is an ordinary conformal Killing vector,

$$
\partial^{\hat{a}} \xi^{\hat{b}}+\partial^{\hat{b}} \xi^{\hat{a}}=\frac{2}{5} \eta^{\hat{a} \hat{b}} \partial_{\hat{c}} \xi^{\hat{c}}
$$

depending parametrically on the Grassmann superspace coordinates,

$$
\xi^{\hat{a}}(x, \theta)=b^{\hat{a}}(\theta)+2 \sigma(\theta) x^{\hat{a}}+\omega^{\hat{a}}{ }_{\hat{b}}(\theta) x^{\hat{b}}+k^{\hat{a}}(\theta) x^{2}-2 x^{\hat{a}} x_{\hat{b}} k^{\hat{b}}(\theta),
$$

with $\omega^{\hat{a} \hat{b}}=-\omega^{\hat{a} \hat{b}}$.

From (5.4) one can derive a closed equation on the vector components $\xi_{\hat{\beta} \hat{\gamma}}=\left(\Gamma^{\hat{b}}\right)_{\hat{\beta} \hat{\gamma}} \xi_{\hat{b}}$ :

$$
D_{(\hat{\alpha}}^{i} \xi_{\hat{\beta}) \hat{\gamma}}=-\frac{1}{5} D^{\hat{\delta} i} \xi_{\hat{\delta}(\hat{\alpha}} \varepsilon_{\hat{\beta}) \hat{\gamma}}
$$

One can also deduce closed equations on the spinor components $\xi_{i}^{\hat{\alpha}}$ :

$$
\begin{aligned}
D_{\hat{\alpha}}^{(i} \xi_{\hat{\beta}}^{j)} & =\frac{1}{4} \varepsilon_{\hat{\alpha} \hat{\beta}} D^{\hat{\gamma}(i} \xi_{\hat{\gamma}}^{j)}, \\
\left(\Gamma^{\hat{b}}\right)_{\hat{\alpha} \hat{\beta}} D^{\hat{\alpha} i} \xi_{i}^{\hat{\beta}} & =0 .
\end{aligned}
$$


At this stage it is useful to let harmonics $u_{i}^{ \pm}$, such that $u^{+i} u_{i}^{-} \neq 0$, enter the scene for the first time. With the definitions $D_{\hat{\alpha}}^{ \pm}=D_{\hat{\alpha}}^{i} u_{i}^{ \pm}$and $\xi_{\hat{\alpha}}^{ \pm}=\xi_{\hat{\alpha}}^{i} u_{i}^{ \pm}$, eq. (5.9) is equivalent to

$$
D_{\hat{\alpha}}^{+} \xi_{\hat{\beta}}^{+}=\frac{1}{4} \varepsilon_{\hat{\alpha} \hat{\beta}} D^{+\hat{\gamma}} \xi_{\hat{\gamma}}^{+} \quad \Longrightarrow \quad D_{\hat{\alpha}}^{+} D_{\hat{\beta}}^{+} \xi_{\hat{\gamma}}^{+}=0
$$

The above results lead to

$$
\left[\xi, D_{\hat{\alpha}}^{i}\right]=\tilde{\omega}_{\hat{\alpha}}^{\hat{\beta}} D_{\hat{\beta}}^{i}-\tilde{\sigma} D_{\hat{\alpha}}^{i}-\tilde{\Lambda}_{j}^{i} D_{\hat{\alpha}}^{j},
$$

where

$$
\tilde{\omega}^{\hat{\alpha} \hat{\beta}}=-\frac{1}{2} D^{k(\hat{\alpha}} \xi_{k}^{\hat{\beta})}, \quad \tilde{\sigma}=\frac{1}{8} D_{\hat{\gamma}}^{k} \xi_{k}^{\hat{\gamma}}, \quad \tilde{\Lambda}^{i j}=\frac{1}{4} D_{\hat{\gamma}}^{(i} \xi^{j) \hat{\gamma}}
$$

The parameters on the right of (5.12) are related to each other as follows

$$
\begin{aligned}
& D_{\hat{\alpha}}^{i} \tilde{\omega}_{\hat{\beta} \hat{\gamma}}=2\left(\varepsilon_{\hat{\alpha} \hat{\beta}} D_{\hat{\gamma}}^{i} \tilde{\sigma}+\varepsilon_{\hat{\alpha} \hat{\gamma}} D_{\hat{\beta}}^{i} \tilde{\sigma}\right), \\
& D_{\hat{\alpha}}^{i} \tilde{\Lambda}^{j k}=3\left(\epsilon^{i k} D_{\hat{\alpha}}^{j} \tilde{\sigma}+\epsilon^{i j} D_{\hat{\alpha}}^{k} \tilde{\sigma}\right) .
\end{aligned}
$$

The superconformal transformation of the superspace integration measure involves

$$
\partial_{\hat{a}} \xi^{\hat{a}}-D_{\hat{\alpha}}^{i} \xi_{i}^{\hat{\alpha}}=2 \tilde{\sigma} .
$$

\subsection{Primary superfields}

Here we give a few examples of 5D primary superfields, without Lorentz indices.

Consider a completely symmetric iso-tensor superfield $H^{i_{1} \ldots i_{n}}=H^{\left(i_{1} \ldots i_{n}\right)}$ with the superconformal transformation law

$$
\delta H^{i_{1} \ldots i_{n}}=-\xi H^{i_{1} \ldots i_{n}}-p \tilde{\sigma} H^{i_{1} \ldots i_{n}}-\tilde{\Lambda}_{k}{ }^{\left(i_{1}\right.} H^{\left.i_{2} \ldots i_{n}\right) k},
$$

with $p$ a constant parameter being equal to half the conformal weight of $H^{i_{1} \ldots i_{n}}$. It turns out that this parameter is equal to $3 n$ if $H^{i_{1} \ldots i_{n}}$ is constrained by

$$
D_{\hat{\alpha}}{ }^{(j} H^{\left.i_{1} \ldots i_{n}\right)}=0 \quad \longrightarrow \quad p=3 n .
$$

The vector multiplet strength transforms as

$$
\delta W=-\xi W-2 \tilde{\sigma} W .
$$

The conformal weight of $W$ is uniquely fixed by the Bianchi identity

$$
D_{\hat{\alpha}}^{(i} D_{\hat{\beta}}^{j)} W=\frac{1}{4} \varepsilon_{\hat{\alpha} \hat{\beta}} D^{\hat{\gamma}(i} D_{\hat{\gamma}}^{j)} W
$$

obeyed by $W$. 


\subsection{Analytic building blocks}

In what follows we make use of the harmonics $u_{i}^{ \pm}$subject to eq. (4.11). As in the $4 \mathrm{D} \mathcal{N}=2$ case, eq. (4.11) has no intrinsic significance, with the only essential condition being $\left(u^{+} u^{-}\right) \equiv u^{+i} u_{i}^{-} \neq 0$. Eq. (4.11) is nevertheless handy, for it allows one to get rid of numerous annoying factors of $\left(u^{+} u^{-}\right)$.

Introduce

$$
\Sigma=\tilde{\Lambda}^{i j} u_{i}^{+} u_{j}^{-}+3 \tilde{\sigma}, \quad \tilde{\Lambda}^{++}=D^{++} \Sigma=\tilde{\Lambda}^{i j} u_{i}^{+} u_{j}^{+} .
$$

It follows from (5.14) and the identity $\left[D^{++}, D_{\hat{\alpha}}^{+}\right]=0$, that $\Sigma$ and $\tilde{\Lambda}^{++}$are analytic superfields,

$$
D_{\hat{\alpha}}^{+} \Sigma=0, \quad D_{\hat{\alpha}}^{+} \tilde{\Lambda}^{++}=0 .
$$

Representing $\xi=\xi^{\hat{a}} \partial_{\hat{a}}-\xi^{+\hat{\alpha}} D_{\hat{\alpha}}^{-}+\xi^{-\hat{\alpha}} D_{\hat{\alpha}}^{+}$, one can now check that

$$
\left[\xi-\tilde{\Lambda}^{++} D^{--}, D_{\hat{\alpha}}^{+}\right]=\tilde{\omega}_{\hat{\alpha}}^{\hat{\beta}} D_{\hat{\beta}}^{+}-(\Sigma-2 \tilde{\sigma}) D_{\hat{\alpha}}^{+} .
$$

This relation implies that the operator $\xi-\tilde{\Lambda}^{++} D^{--}$maps every analytic superfield into an analytic one. It is worth pointing out that the superconformal transformation of the analytic subspace measure involves

$$
\partial_{\hat{a}} \xi^{\hat{a}}+D_{\hat{\alpha}}^{-} \xi^{+\hat{\alpha}}-D^{--} \tilde{\Lambda}^{++}=2 \Sigma .
$$

\subsection{Harmonic superconformal multiplets}

We present here several superconformal multiplets that are globally defined over the harmonic superspace. Such a multiplet is described by a smooth Grassmann analytic superfields $\Phi_{\kappa}^{(n)}\left(z, u^{+}, u^{-}\right)$,

$$
D_{\hat{\alpha}}^{+} \Phi_{\kappa}^{(n)}=0
$$

which is endowed with the following superconformal transformation law

$$
\delta \Phi_{\kappa}^{(n)}=-\left(\xi-\tilde{\Lambda}^{++} D^{--}\right) \Phi_{\kappa}^{(n)}-\kappa \Sigma \Phi_{\kappa}^{(n)} .
$$

The parameter $\kappa$ is related to the conformal weight of $\Phi_{\kappa}^{(n)}$. We will call $\Phi_{\kappa}^{(n)}$ an analytic density of weight $\kappa$. When $n$ is even, one can define real superfields, $\breve{\Phi}_{\kappa}^{(n)}=\Phi_{\kappa}^{(n)}$, with respect to the analyticity-preserving conjugation [5, 6] (also known as 'smile-conjugation').

Let $V^{++}$be a real analytic gauge potential describing a $U(1)$ vector multiplet. Its superconformal transformation is

$$
\delta V^{++}=-\left(\xi-\tilde{\Lambda}^{++} D^{--}\right) V^{++} .
$$


Associated with the gauge potential is the field strength

$$
W=\frac{\mathrm{i}}{8} \int \mathrm{d} u\left(\hat{D}^{-}\right)^{2} V^{++}, \quad\left(\hat{D}^{ \pm}\right)^{2}=D^{ \pm \hat{\alpha}} D_{\hat{\alpha}}^{ \pm}
$$

which is known to be invariant under the gauge transformation $\delta V^{++}=D^{++} \lambda$, where the gauge parameter $\lambda$ is a real analytic superfield. The superconformal transformation of $W$,

$$
\delta W=-\frac{\mathrm{i}}{8} \int \mathrm{d} u\left(\hat{D}^{-}\right)^{2}\left(\xi+\left(D^{--} \tilde{\Lambda}^{++}\right)\right) V^{++}
$$

can be shown to coincide with (5.18).

There are many ways to describe a hypermultiplet. In particular, one can use an analytic superfield $q^{+}(z, u)$ and its smile-conjugate $\breve{q}^{+}(z, u)[5,6]$. They transform as follows:

$$
\delta q^{+}=-\left(\xi-\tilde{\Lambda}^{++} D^{--}\right) q^{+}-\Sigma q^{+}, \quad \delta \breve{q}^{+}=-\left(\xi-\tilde{\Lambda}^{++} D^{--}\right) \breve{q}^{+}-\Sigma \breve{q}^{+} .
$$

One has $\kappa=n$ in $(5.25)$, if the superfield is annihilated by $D^{++}$,

$$
\begin{gathered}
D_{\hat{\alpha}}^{+} H^{(n)}=D^{++} H^{(n)}=0 \quad \longrightarrow \quad H^{(n)}(z, u)=H^{i_{1} \ldots i_{n}}(z) u_{i_{1}}^{+} \ldots u_{i_{n}}^{+}, \\
\delta H^{(n)}=-\left(\xi-\tilde{\Lambda}^{++} D^{--}\right) H^{(n)}-n \Sigma H^{(n)} .
\end{gathered}
$$

Here the harmonic-independent superfield $H^{i_{1} \ldots i_{n}}$ transforms according to (5.16) with $p=3 n$.

\subsection{Projective superconformal multiplets}

In the projective superspace approach, one deals only with superfields $\phi^{(n)}\left(z, u^{+}\right)$ obeying the constraints

$$
D_{\hat{\alpha}}^{+} \boldsymbol{\phi}^{(n)}=D^{++} \boldsymbol{\phi}^{(n)}=0, \quad n \geq 0
$$

Here the first constraint means that $\phi^{(n)}$ is Grassmann analytic, while the second constraint demands independence of $u^{-}$. Unlike the harmonic superspace approach, however, $\phi^{(n)}\left(z, u^{+}\right)$is not required to be well-defined over the two-sphere, that is, $\boldsymbol{\phi}^{(n)}$ may have singularities (say, poles) at some points of $S^{2}$. The presence of singularities turns out to be perfectly $\mathrm{OK}$ since the projective-superspace action involves a contour integral in $S^{2}$, see below. 
We assume that $\boldsymbol{\phi}^{(p)}(z, u)$ is non-singular outside the north and south poles of $S^{2}$. In the north chart, we can represent

$$
D_{\hat{\alpha}}^{+}=-u^{+\underline{1}} \nabla_{\hat{\alpha}}(w), \quad \nabla_{\hat{\alpha}}(w)=-D_{\hat{\alpha}}^{i} w_{i}, \quad w_{i}=(-w, 1)
$$

Then, the equations (5.31) are equivalent to

$$
\phi(z, w)=\sum_{n=-\infty}^{+\infty} \phi_{n}(z) w^{n}, \quad \nabla_{\hat{\alpha}}(w) \phi(z, w)=0
$$

with the holomorphic superfield $\phi(z, w) \propto \phi^{(n)}\left(z, u^{+}\right)$. These relations define a projective multiplet, following the four-dimensional terminology [9]. Associated with $\phi(z, w)$ is its smile-conjugate

$$
\breve{\phi}(z, w)=\sum_{n=-\infty}^{+\infty}(-1)^{n} \bar{\phi}_{-n}(z) w^{n}, \quad \nabla_{\hat{\alpha}}(w) \breve{\phi}(z, w)=0
$$

which is also a projective multiplet. If $\breve{\phi}(z, w)=\phi(z, w)$, the projective superfield is called real.

Below we present several superconformal multiplets as defined in the north chart. The corresponding transformations laws involve the two analytic building blocks:

$\tilde{\Lambda}^{++}(w)=\tilde{\Lambda}^{i j} w_{i}^{+} w_{j}^{+}=\tilde{\Lambda} \underline{11} w^{2}-2 \tilde{\Lambda}^{12} w+\tilde{\Lambda}^{\underline{22}}, \quad \Sigma(w)=\tilde{\Lambda}^{\underline{1}} w_{i}+3 \tilde{\sigma}=-\tilde{\Lambda}^{\underline{11}} w+\tilde{\Lambda} \underline{12}+3 \tilde{\sigma}$.

Similar structures occur in the south chart, that is

$$
\tilde{\Lambda}^{++}(y)=\tilde{\Lambda}^{i j} y_{i}^{+} y_{j}^{+}=\tilde{\Lambda} \underline{11}-2 \tilde{\Lambda}^{\underline{12}} y+\tilde{\Lambda}^{\underline{22}} y^{2}, \quad \Sigma(y)=\tilde{\Lambda}^{\underline{2 i}} y_{i}+3 \tilde{\sigma}=-\tilde{\Lambda}^{\underline{12}}+\tilde{\Lambda}^{\underline{22}} y+3 \tilde{\sigma} .
$$

In the overlap of the two charts, we have

$$
\begin{aligned}
\tilde{\Lambda}^{++}(y) & =\frac{1}{w^{2}} \tilde{\Lambda}^{++}(w) \quad \longrightarrow \quad \tilde{\Lambda}^{++}(y) \partial_{y}=-\tilde{\Lambda}^{++}(w) \partial_{w} \\
\Sigma(y) & =\Sigma(w)+\frac{1}{w} \tilde{\Lambda}^{++}(w) .
\end{aligned}
$$

To realise a massless vector multiplet, one uses the so-called tropical multiplet described by

$$
V(z, w)=\sum_{n=-\infty}^{+\infty} V_{n}(z) w^{n}, \quad \bar{V}_{n}=(-1)^{n} V_{-n} .
$$

Its superconformal transformation

$$
\delta V=-\left(\xi+\tilde{\Lambda}^{++}(w) \partial_{w}\right) V .
$$


The field strength of the vector multiplet ${ }^{7}$ is

$$
W(z)=-\frac{1}{16 \pi \mathrm{i}} \oint \mathrm{d} w\left(\hat{D}^{-}\right)^{2} V(z, w)=\frac{1}{4 \pi \mathrm{i}} \oint \frac{\mathrm{d} w}{w} \mathcal{P}(w) V(z, w)
$$

where

$$
\mathcal{P}(w)=\frac{1}{4 w}\left(\bar{D}_{\underline{1}}\right)^{2}+\partial_{5}-\frac{w}{4}\left(D^{\underline{1}}\right)^{2} .
$$

The superconformal transformation of $W$ can be shown to coincide with (5.18). The field strength (5.38) is invariant under the gauge transformation

$$
\delta V(z, w)=\mathrm{i}(\breve{\lambda}(z, w)-\lambda(z, w))
$$

with $\lambda(z, w)$ an arbitrary arctic multiplet, see below.

To describe a massless off-shell hypermultiplet, one can use the so-called arctic multiplet $\Upsilon(z, w)$ :

$$
\boldsymbol{q}^{+}(z, u)=u^{+\underline{1}} \Upsilon(z, w) \sim \Upsilon(z, w), \quad \Upsilon(z, w)=\sum_{n=0}^{\infty} \Upsilon_{n}(z) w^{n}
$$

The smile-conjugation of $\boldsymbol{q}^{+}$leads to the so-called the antarctic multiplet $\breve{\Upsilon}(z, w)$ :

$$
\breve{\boldsymbol{q}}^{+}(z, u)=u^{+\underline{2}} \breve{\Upsilon}(z, w) \sim w \breve{\Upsilon}(z, w) \quad \breve{\Upsilon}(z, w)=\sum_{n=0}^{\infty}(-1)^{n} \bar{\Upsilon}_{n}(z) \frac{1}{w^{n}}
$$

Their superconformal transformations are

$$
\begin{gathered}
\delta \Upsilon=-\left(\xi+\tilde{\Lambda}^{++}(w) \partial_{w}\right) \Upsilon-\Sigma(w) \Upsilon, \\
\delta \breve{\Upsilon}=-\frac{1}{w}\left(\xi+\tilde{\Lambda}^{++}(w) \partial_{w}\right)(w \breve{\Upsilon})-\Sigma(w) \breve{\Upsilon} .
\end{gathered}
$$

In the south chart, these transformations take the form

$$
\begin{gathered}
\delta \Upsilon=-\frac{1}{y}\left(\xi-\tilde{\Lambda}^{++}(y) \partial_{y}\right)(y \Upsilon)-\Sigma(y) \Upsilon \\
\delta \breve{\Upsilon}=-\left(\xi-\tilde{\Lambda}^{++}(y) \partial_{y}\right) \breve{\Upsilon}-\Sigma(y) \breve{\Upsilon} .
\end{gathered}
$$

Both $\Upsilon(z, w)$ and $\breve{\Upsilon}(z, w)$ constitute the so-called polar multiplet.

\footnotetext{
${ }^{7} \mathrm{~A}$ more general form for the field strength (5.38) is given in Appendix B.
} 
Since the product of two arctic superfields is again arctic, from (5.43) we obtain more general transformation laws

$$
\begin{gathered}
\delta \Upsilon_{\kappa}=-\left(\xi+\tilde{\Lambda}^{++}(w) \partial_{w}\right) \Upsilon_{\kappa}-\kappa \Sigma(w) \Upsilon_{\kappa}, \\
\delta \breve{\Upsilon}_{\kappa}=-\frac{1}{w^{\kappa}}\left(\xi+\tilde{\Lambda}^{++}(w) \partial_{w}\right)\left(w^{\kappa} \breve{\Upsilon}_{\kappa}\right)-\kappa \Sigma(w) \breve{\Upsilon}_{\kappa},
\end{gathered}
$$

for some parameter $\kappa$. The case $\kappa=1$ corresponds to free hypermultiplet dynamics, see below.

Since the product $U_{\kappa}=\breve{\Upsilon}_{\kappa} \Upsilon_{\kappa}$ is a tropical multiplet, we obtain more general transformation laws than the one defined by eq. (5.37):

$$
\delta U_{\kappa}=-\frac{1}{w^{\kappa}}\left(\xi+\tilde{\Lambda}^{++}(w) \partial_{w}\right)\left(w^{\kappa} U_{\kappa}\right)-2 \kappa \Sigma(w) U_{\kappa}
$$

Finally, let us consider the projective-superspace reformulation of the multiplets (5.30) with an even superscript,

$$
\begin{aligned}
& H^{(2 n)}(z, u)=\left(\mathrm{i} u^{+1} u^{+2}\right)^{n} H^{[2 n]}(z, w) \sim(\mathrm{i} w)^{n} H^{[2 n]}(z, w), \\
& H^{[2 n]}(z, w)=\sum_{k=-n}^{n} H_{k}(z) w^{n}, \quad \bar{H}_{k}=(-1)^{k} H_{-k} .
\end{aligned}
$$

The projective superfield $H^{[2 n]}(z, w)$ is often called a real $O(2 n)$ multiplet [9]. Its superconformal transformation in the north chart is

$$
\delta H^{[2 n]}=-\frac{1}{w^{n}}\left(\xi+\tilde{\Lambda}^{++}(w) \partial_{w}\right)\left(w^{n} H^{[2 n]}\right)-2 n \Sigma(w) H^{[2 n]} .
$$

In a similar way one can introduce complex $O(2 n+1)$ multiplets. In what follows, we will use the same name ' $O(n)$ multiplet' for both harmonic multiplets (5.30) and the projective ones just introduced.

Among the projective superconformal multiplets considered, it is only the $O(n)$ multiplets which can be lifted to well-defined representations of the superconformal group on a compactified 5D harmonic superspace. The other multiplets realise the superconformal algebra only.

\section{5D superconformal theories}

With the tools developed, we are prepared to constructing 5D superconformal theories. Superfield formulations for $5 \mathrm{D} \mathcal{N}=1$ rigid supersymmetric theories were earlier 
elaborated in the harmonic $[47,15]$ and projective $[15]$ superspace settings. $^{8}$

\subsection{Models in harmonic superspace}

Let $\mathcal{L}^{(+4)}$ be an analytic density of weight +2 . Its superconformal transformation is a total derivative,

$$
\begin{aligned}
\delta \mathcal{L}^{(+4)} & =-\left(\xi-\tilde{\Lambda}^{++} D^{--}\right) \mathcal{L}^{(+4)}-2 \Sigma \mathcal{L}^{(+4)} \\
& =-\partial_{\hat{a}}\left(\xi^{\hat{a}} \mathcal{L}^{(+4)}\right)-D_{\hat{\alpha}}^{-}\left(\xi^{+\hat{\alpha}} \mathcal{L}^{(+4)}\right)+D^{--}\left(\tilde{\Lambda}^{++} \mathcal{L}^{(+4)}\right)
\end{aligned}
$$

Therefore, such a superfield generates a superconformal invariant of the form

$$
\int \mathrm{d} \zeta^{(-4)} \mathcal{L}^{(+4)}
$$

where

$$
\int \mathrm{d} \zeta^{(-4)}:=\int \mathrm{d} u \int \mathrm{d}^{5} x\left(\hat{D}^{-}\right)^{4}, \quad\left(\hat{D}^{ \pm}\right)^{4}=-\frac{1}{32}\left(\hat{D}^{ \pm}\right)^{2}\left(\hat{D}^{ \pm}\right)^{2}
$$

This is the harmonic superspace action [6] as applied to the five-dimensional case.

Let $V^{++}$be the gauge potential of an Abelian vector multiplet. Given a real $O(2)$ multiplet $\mathcal{L}^{++}$,

$$
D_{\hat{\alpha}}^{+} \mathcal{L}^{++}=D^{++} \mathcal{L}^{++}=0, \quad \delta \mathcal{L}^{++}=-\left(\xi-\tilde{\Lambda}^{++} D^{--}\right) \mathcal{L}^{++}-2 \Sigma \mathcal{L}^{++}
$$

we can generate the following superconformal invariant

$$
\int \mathrm{d} \zeta^{(-4)} V^{++} \mathcal{L}^{++}
$$

Because of the constraint $D^{++} \mathcal{L}^{++}=0$, the integral is invariant under the vector multiplet gauge transformation $\delta V^{++}=-D^{++} \lambda$, with $\lambda$ a real analytic gauge parameter.

The field strength of the vector multiplet, $W$, is a primary superfield with the transformation (5.18). Using $W$, one can construct the following analytic superfield [15]

$$
-\mathrm{i} G^{++}=D^{+\hat{\alpha}} W D_{\hat{\alpha}}^{+} W+\frac{1}{2} W\left(\hat{D}^{+}\right)^{2} W, \quad D_{\hat{\alpha}}^{+} G^{++}=D^{++} G^{++}=0 .
$$

which transforms as a harmonic superfield weight 2 ,

$$
\delta G^{++}=-\left(\xi-\tilde{\Lambda}^{++} D^{--}\right) G^{++}-2 \Sigma G^{++} .
$$

\footnotetext{
${ }^{8}$ In the case of $6 \mathrm{D} \mathcal{N}=(1,0)$ rigid supersymmetric theories, superfield formulations have been developed in the conventional [48], harmonic [49] and projective [50] superspace settings.
} 
In other words, $G^{++}$is a real $O(2)$ multiplet. As a result, the supersymmetric ChernSimons action ${ }^{9}[15]$

$$
S_{\mathrm{CS}}\left[V^{++}\right]=\frac{1}{12} \int \mathrm{d} \zeta^{(-4)} V^{++} G^{++}
$$

is superconformally invariant.

Super Chern-Simons theory (6.8) is quite remarkable as compared with the superconformal models of a single vector multiplet in four and six dimensions. In the $4 \mathrm{D} \mathcal{N}=2$ case, the analogue of $G^{++}$in (6.8) is known to be $D^{+\alpha} D_{\alpha}^{+} W=\bar{D}_{\dot{\alpha}}^{+} \bar{D}^{+\dot{\alpha}} \bar{W}$, with $W$ the chiral field strength, and therefore the model is free. In the case $6 \mathrm{D} \mathcal{N}=(1,0)$, the analogue of $G^{++}$in $(6.8)$ is $\left(D^{+}\right)^{4} D_{\hat{\alpha}}^{-} W^{-\hat{\alpha}}$, see [18] for more details, and therefore the models is not only free but also has higher derivatives. It is only in five dimensions that the requirement of superconformal invariance leads to a nontrivial dynamical system.

The model (6.8) admits interesting generalisations. In particular, given several Abelian vector multiplets $V_{I}^{++}$, where $I=1, \ldots, n$, the composite superfield (6.6) is generalised as follows:

$$
\begin{aligned}
G^{++} \rightarrow G_{I J}^{++}=G_{(I J)}^{++} & =\mathrm{i}\left\{D^{+\hat{\alpha}} W_{I} D_{\hat{\alpha}}^{+} W_{J}+\frac{1}{2} W_{(I}\left(\hat{D}^{+}\right)^{2} W_{J)}\right\}, \\
D_{\hat{\alpha}}^{+} G_{I J}^{++} & =D^{++} G_{I J}^{++}=0 .
\end{aligned}
$$

The gauge-invariant and superconformal action (6.8) turns into

$$
\tilde{S}_{\mathrm{CS}}=\frac{1}{12} \int \mathrm{d} \zeta^{(-4)} V_{I}^{++} c_{I, J K} G_{J K}^{++}, \quad c_{I, J K}=c_{I, K J}
$$

for some constant parameters $c_{I, J K}$. One can also generalise the super Chern-Simons theory (6.8) to the non-Abelian case.

In harmonic superspace, some superconformal transformation laws are effectively independent (if properly understood) of the dimension of space-time. As a result, some 4D $\mathcal{N}=2$ superconformal theories can be trivially extended to five dimensions. In particular, the model for a massless $U(1)$ charged hypermultiplet [5]

$$
S_{\text {hyper }}=-\int \mathrm{d} \zeta^{(-4)} \breve{q}^{+}\left(D^{++}+\mathrm{i} e V^{++}\right) q^{+}
$$

can be seen to be superconformal. This follows from eqs. (5.26) and (5.29), in conjunction with the observation that the transformation laws of $q^{+}$and $D^{++} q^{+}$are identical.

\footnotetext{
${ }^{9} \mathrm{~A}$ different form for this action was given in [47].
} 
The dynamical system $S_{\mathrm{CS}}+S_{\text {hyper }}$ can be chosen to describe the supergravity compensator sector (vector multiplet plus hypermultiplet) when describing 5D simple supergravity within the superconformal tensor calculus $[3,4]$. Then, the hypermultiplet charge $e$ is equivalent to the presence of a non-vanishing cosmological constant, similar to the $4 \mathrm{D} \mathcal{N}=2$ case $[6]$.

Our next example is a naive $5 \mathrm{D}$ generalisation of the $4 \mathrm{D} \mathcal{N}=2$ improved tensor multiplet $[51,52,7]$ which was described in the harmonic superspace approach in $[53,6]$. Let us consider the action

$$
S_{\text {tensor }}\left[H^{++}\right]=\int \mathrm{d} \zeta^{(-4)} \mathcal{L}^{(+4)}\left(H^{++}, u\right)
$$

where

$$
\mathcal{L}^{(+4)}\left(H^{++}, u\right)=\mu^{3}\left(\frac{\mathcal{H}^{++}}{1+\sqrt{1+\mathcal{H}^{++} c^{--}}}\right)^{2}, \quad \mathcal{H}^{++}=H^{++}-c^{++}
$$

with $\mu$ a constant parameter of unit mass dimension, and $c^{++}$a (space-time) independent holomorphic vector field on $S^{2}$,

$$
c^{ \pm \pm}(u)=c^{i j} u_{i}^{ \pm} u_{j}^{ \pm}, \quad c^{i j} c_{i j}=2, \quad c^{i j}=\text { const }
$$

Here $H^{++}(z, u)$ is a real $O(2)$ multiplet possessing the superconformal transformation law (5.30) with $n=2$. The superconformal invariance of (6.12) can be proved in complete analogy to the detailed consideration given $[53,6]$.

Now, let us couple the vector multiplet to the real $O(2)$ multiplet by putting forward the action

$$
S_{\text {vector-tensor }}\left[V^{++}, H^{++}\right]=S_{\mathrm{CS}}\left[V^{++}\right]+\kappa \int \mathrm{d} \zeta^{(-4)} V^{++} H^{++}+S_{\text {tensor }}\left[H^{++}\right],
$$

with $\kappa$ a coupling constant. This action is both gauge-invariant and superconformal. It is a five-dimensional generalisation of the $4 \mathrm{D} \mathcal{N}=2$ model for massive tensor multiplet introduced in [54].

The dynamical system $S_{\text {vector-tensor }}$ can be chosen to describe the supergravity compensator sector (vector multiplet plus tensor multiplet) when describing 5D simple supergravity within the superconformal tensor calculus [3, 4]. Then, the coupling constant $\kappa$ is equivalent to a cosmological constant, similar to the $4 \mathrm{D} \mathcal{N}=2$ case [10].

Finally, consider the vector multiplet model

$$
S_{\mathrm{CS}}\left[V^{++}\right]+S_{\text {tensor }}\left[G^{++} / \mu^{3}\right]
$$


with $G^{++}$the composite superfield (6.6). The second term here turns out to be a unique superconformal extension of the $F^{4}$-term, where $F$ is the field strength of the component gauge field. In this respect, it is instructive to recall its $4 \mathrm{D} \mathcal{N}=2$ analogue [55]

$$
\int \mathrm{d}^{4} x \mathrm{~d}^{8} \theta \ln W \ln \bar{W}
$$

The latter can be shown [56] to be a unique $\mathcal{N}=2$ superconformal invariant in the family of actions of the form $\int \mathrm{d}^{4} x \mathrm{~d}^{8} \theta H(W, \bar{W})$ introduced for the first time in [57]. In five space-time dimensions, if one looks for a superconformal invariant of the form $\int \mathrm{d}^{5} x \mathrm{~d}^{8} \theta H(W)$, the general solution is $H(W) \propto W$, as follows from (5.15) and (5.18), and this choice corresponds to a total derivative.

\subsection{Models in projective superspace}

Let $\mathcal{L}(z, w)$ be an analytic superfield transforming according to (5.46) with $\kappa=1$. This transformation law can be rewritten as

$$
\begin{aligned}
w \delta \mathcal{L} & =-\left(\xi+\tilde{\Lambda}^{++} \partial_{w}\right)(w \mathcal{L})-2 w \Sigma \mathcal{L} \\
& =-\partial_{\hat{a}}\left(\xi^{\hat{a}} w \mathcal{L}\right)-D_{\hat{\alpha}}^{-}\left(\xi^{+\hat{\alpha}} w \mathcal{L}\right)-\partial_{w}\left(\tilde{\Lambda}^{++} w \mathcal{L}\right) .
\end{aligned}
$$

Such a superfield turns out to generate a superconformal invariant of the form

$$
I=\oint \frac{\mathrm{d} w}{2 \pi \mathrm{i}} \int \mathrm{d}^{5} x\left(\hat{D}^{-}\right)^{4} w \mathcal{L}(z, w)
$$

where $\oint \mathrm{d} w$ is a (model-dependent) contour integral in $\mathbb{C} P^{1}$. Indeed, it follows from (6.18) that this functional does not change under the superconformal transformations. Eq. (6.19) generalises the projective superspace action $[7,8]$ to the five-dimensional case. A more general form for this action, which does not imply the projective gauge conditions (4.23) and is based on the construction in [8], is given in Appendix B.

It is possible to bring the action (6.19) to a somewhat simpler form if one exploits the fact that $\mathcal{L}$ is Grassmann analytic. Using the considerations outlined in Appendix $\mathrm{C}$ gives

$$
\int \mathrm{d}^{5} x\left(\hat{D}^{-}\right)^{4} \mathcal{L}=\frac{1}{w^{2}} \int \mathrm{d}^{5} x D^{4} \mathcal{L}\left|, \quad D^{4}=\frac{1}{16}\left(D^{\underline{1}}\right)^{2}\left(\bar{D}_{\underline{1}}\right)^{2}\right| .
$$

Here $D^{4}$ is the Grassmann part of the integration measure of $4 \mathrm{D} \mathcal{N}=1$ superspace, $\int \mathrm{d}^{4} \theta=D^{4}$. Then, functional (6.19) turns into

$$
I=\oint \frac{\mathrm{d} w}{2 \pi \mathrm{i} w} \int \mathrm{d}^{5} x D^{4} \mathcal{L}=\oint \frac{\mathrm{d} w}{2 \pi \mathrm{i} w} \int \mathrm{d}^{5} x \mathrm{~d}^{4} \theta \mathcal{L} .
$$


Our first example is the tropical multiplet formulation for the super Chern-Simons theory $[15]$

$$
S_{\mathrm{CS}}=-\frac{1}{12} \oint \frac{\mathrm{d} w}{2 \pi \mathrm{i} w} \int \mathrm{d}^{5} x \mathrm{~d}^{4} \theta V G
$$

with the contour around the origin. Here $G(w)$ is the composite $O(2)$ multiplet (6.6) constructed out of the tropical gauge potential $V(w)$,

$$
G^{++}=\left(\mathrm{i} u^{+1} u^{+\underline{2}}\right) G(w) \sim \mathrm{i} w G(w), \quad G(w)=-\frac{1}{w} \Psi+K+w \bar{\Psi}
$$

The explicit expressions for the superfields $\Psi$ and $K$ can be found in [15]. The above consideration and the transformation laws (5.37) and (6.7) imply that the action (6.22) is superconformal.

Next, let us generalise to five dimensions the charged $\Upsilon$-hypermultiplet model of [9]:

$$
S_{\text {hyper }}=\oint \frac{\mathrm{d} w}{2 \pi \mathrm{i} w} \int \mathrm{d}^{5} x \mathrm{~d}^{4} \theta \breve{\Upsilon} \mathrm{e}^{q V} \Upsilon
$$

with $q$ the hypermultiplet charge, and the integration contour around the origin. This action is superconformal, in accordance with the transformation laws (5.37) and (5.43). It is also invariant under gauge transformations

$$
\delta \Upsilon=\mathrm{i} q \Upsilon, \quad \delta V=\mathrm{i}(\breve{\lambda}-\lambda)
$$

with $\lambda$ an arctic superfield.

Now, let us couple the vector multiplet to a real $O(2)$ multiplet $H(w)$

$$
H^{++}=\left(\mathrm{i} u^{+1} u^{+2}\right) H(w) \sim \mathrm{i} w H(w), \quad H(w)=-\frac{1}{w} \Phi+L+w \bar{\Phi}
$$

We introduce the vector-tensor system

$$
S=-\oint \frac{\mathrm{d} w}{2 \pi \mathrm{i} w} \int \mathrm{d}^{5} x \mathrm{~d}^{4} \theta V\left\{\frac{1}{12} G+\kappa H\right\}+\mu^{3} \oint \frac{\mathrm{d} w}{2 \pi \mathrm{i} w} \int \mathrm{d}^{5} x \mathrm{~d}^{4} \theta H \ln H
$$

where the first term on the right involves a contour around the origin, while the second comes with a contour turning clockwise and anticlockwise around the roots of of the quadratic equation $w H(w)=0$. The second term in (6.27) is a minimal 5D extension of the $4 \mathrm{D} \mathcal{N}=2$ improved tensor multiplet [7]. It should be pointed out that the component superfields in (6.26) obey the constraints [15]

$$
\bar{D}^{\dot{\alpha}} \Phi=0, \quad-\frac{1}{4} \bar{D}^{2} L=\partial_{5} \Phi .
$$


It should be also remarked that the real linear superfield $L$ can always be dualised into a chiral scalar and its conjugate [15], which generates a special chiral superpotential.

Given several $O(2)$ multiplets $H^{I}(w)$, where $I=1, \ldots, n$, superconformal dynamics is generated by the action

$$
S=\oint \frac{\mathrm{d} w}{2 \pi \mathrm{i} w} \int \mathrm{d}^{5} x \mathrm{~d}^{4} \theta \mathcal{F}\left(H^{I}\right), \quad I=1, \ldots, n
$$

where $\mathcal{F}(H)$ is a weakly homogeneous function of first degree in the variables $H$,

$$
\oint \frac{\mathrm{d} w}{2 \pi \mathrm{i} w} \int \mathrm{d}^{5} x \mathrm{~d}^{4} \theta\left\{H^{I} \frac{\partial \mathcal{F}(H)}{\partial H^{I}}-\mathcal{F}(H)\right\}=0 .
$$

This is completely analogous to the four-dimensional case $[7,10,16]$ where the component structure of such sigma-models has been studied in detail [60].

A great many superconformal models can be obtained if one considers $\Upsilon$-hypermultiplet actions of the form

$$
S=\oint \frac{\mathrm{d} w}{2 \pi \mathrm{i} w} \int \mathrm{d}^{5} x \mathrm{~d}^{4} \theta K\left(\Upsilon^{I}, \breve{\Upsilon}^{\bar{J}}\right), \quad I, \bar{J}=1, \ldots, n
$$

with the contour around the origin. Let us first assume that the superconformal transformations of all $\Upsilon$ 's and $\breve{\Upsilon}$ 's have the form (5.43). Then, in accordance with general principles, the action is superconformal if $K(\Upsilon, \breve{\Upsilon})$ is a weakly homogeneous function of first degree in the variables $\Upsilon$,

$$
\oint \frac{\mathrm{d} w}{2 \pi \mathrm{i} w} \int \mathrm{d}^{5} x \mathrm{~d}^{4} \theta\left\{\Upsilon^{I} \frac{\partial K(\Upsilon, \breve{\Upsilon})}{\partial \Upsilon^{I}}-K(\Upsilon, \breve{\Upsilon})\right\}=0
$$

This homogeneity condition is compatible with the Kähler invariance

$$
K(\Upsilon, \breve{\Upsilon}) \quad \longrightarrow \quad K(\Upsilon, \breve{\Upsilon})+\Lambda(\Upsilon)+\bar{\Lambda}(\breve{\Upsilon})
$$

which the model (6.31) possesses [14, 58, 15].

Unlike the $O(n)$ multiplets, the superconformal transformations of $\Upsilon$ and $\breve{\Upsilon}$ are not fixed uniquely by the constraints, as directly follows from (5.45). Therefore, one can consider superconformal sigma-models of the form (6.31) in which the dynamical variables $\Upsilon$ 's consist of several subsets with different values for the weight $\kappa$ in (5.45), and then $K(\Upsilon, \breve{\Upsilon})$ should obey weaker conditions than eq. (6.32). Such a situation occurs, for instance, if one starts with a gauged linear sigma-model and then integrates out the gauge multiplet, in the spirit of $[52,16]$. As an example, consider

$$
S=\oint \frac{\mathrm{d} w}{2 \pi \mathrm{i} w} \int \mathrm{d}^{5} x \mathrm{~d}^{4} \theta\left\{\breve{\Upsilon}^{\alpha} \eta_{\alpha \beta} \Upsilon^{\beta} \mathrm{e}^{V}+\breve{\Upsilon}^{\mu} \eta_{\mu \nu} \Upsilon^{\nu} \mathrm{e}^{-V}\right\}
$$


where $\eta_{\alpha \beta}$ and $\eta_{\mu \nu}$ are constant diagonal metrics, $\alpha=1, \ldots, m$ and $\mu=1, \ldots, n$. Integrating out the tropical multiplet gives the gauge-invariant action

$$
S=2 \oint \frac{\mathrm{d} w}{2 \pi \mathrm{i} w} \int \mathrm{d}^{5} x \mathrm{~d}^{4} \theta \sqrt{\breve{\Upsilon}^{\alpha} \eta_{\alpha \beta} \Upsilon^{\beta} \breve{\Upsilon}^{\mu} \eta_{\mu \nu} \Upsilon^{\nu}}
$$

The gauge freedom can be completely fixed by setting, say, one of the superfields $\Upsilon^{\nu}$ to be unity. Then, the action becomes

$$
S=2 \oint \frac{\mathrm{d} w}{2 \pi \mathrm{i} w} \int \mathrm{d}^{5} x \mathrm{~d}^{4} \theta \sqrt{\breve{\Upsilon}^{\alpha} \eta_{\alpha \beta} \Upsilon^{\beta}\left(\eta_{n n}+\breve{\Upsilon} \underline{\underline{\mu}} \eta_{\underline{\mu} \underline{\underline{\nu}}} \Upsilon \underline{\nu}\right)},
$$

where $\underline{\mu}, \underline{\nu}=1, \ldots, n-1$. This action is still superconformal, but now $\Upsilon^{\beta}$ and $\Upsilon \underline{\nu}$ transform according to (5.45) with $\kappa=2$ and $\kappa=0$, respectively.

Sigma-models (6.31) have an interesting geometric interpretation if $K(\Phi, \bar{\Phi})$ is the Kähler potential of a Kähler manifold $\mathcal{M}[14,58,15]$. Among the component superfields of $\Upsilon(z, w)=\sum_{n=0}^{\infty} \Upsilon_{n}(z) w^{n}$, the leading components $\Phi=\Upsilon_{0} \mid$ and $\Gamma=\Upsilon_{1} \mid$ considered as $4 \mathrm{D} \mathcal{N}=1$ superfields, are constrained:

$$
\bar{D}^{\dot{\alpha}} \Phi=0, \quad-\frac{1}{4} \bar{D}^{2} \Gamma=\partial_{5} \Phi .
$$

The $\Phi$ and $\Gamma$ can be regarded as a complex coordinate of the Kähler manifold and a tangent vector at point $\Phi$ of the same manifold, and therefore they parametrize the tangent bundle $T \mathcal{M}$ of the Kähler manifold. The other components, $\Upsilon_{2}, \Upsilon_{3}, \ldots$, are complex unconstrained superfields. These superfields are auxiliary since they appear in the action without derivatives. The auxiliary superfields $\Upsilon_{2}, \Upsilon_{3}, \ldots$, and their conjugates, can be eliminated with the aid of the corresponding algebraic equations of motion

$$
\oint \mathrm{d} w w^{n-1} \frac{\partial K(\Upsilon, \breve{\Upsilon})}{\partial \Upsilon^{I}}=0, \quad n \geq 2
$$

Their elimination can be carried out using the ansatz

$$
\Upsilon_{n}^{I}=\sum_{p=o}^{\infty} U_{J_{1} \ldots J_{n+p}}^{I} \bar{L}_{1} \ldots \bar{L}_{p}(\Phi, \bar{\Phi}) \Gamma^{J_{1}} \ldots \Gamma^{J_{n+p}} \bar{\Gamma}^{\bar{L}_{1}} \ldots \bar{\Gamma}^{\bar{L}_{p}}, \quad n \geq 2
$$

It can be shown that the coefficient functions $U$ 's are uniquely determined by equations (6.38) in perturbation theory. Upon elimination of the auxiliary superfields, the action (6.31) takes the form

$$
\begin{aligned}
S[\Phi, \bar{\Phi}, \Gamma, \bar{\Gamma}]=\int \mathrm{d}^{5} x \mathrm{~d}^{4} \theta\left\{K(\Phi, \bar{\Phi})-g_{I \bar{J}}(\Phi, \bar{\Phi}) \Gamma^{I} \bar{\Gamma}^{\bar{J}}\right. \\
\left.+\sum_{p=2}^{\infty} \mathcal{R}_{I_{1} \cdots I_{p} \bar{J}_{1} \ldots \bar{J}_{p}}(\Phi, \bar{\Phi}) \Gamma^{I_{1}} \ldots \Gamma^{I_{p}} \bar{\Gamma}^{\bar{J}_{1}} \ldots \bar{\Gamma}^{\bar{J}_{p}}\right\}
\end{aligned}
$$


where the tensors $\mathcal{R}_{I_{1} \cdots I_{p} \bar{J}_{1} \cdots \bar{J}_{p}}$ are functions of the Riemann curvature $R_{I \bar{J} K \bar{L}}(\Phi, \bar{\Phi})$ and its covariant derivatives. Each term in the action contains equal powers of $\Gamma$ and $\bar{\Gamma}$, since the original model (6.31) is invariant under rigid $U(1)$ transformations

$$
\Upsilon(w) \mapsto \Upsilon\left(\mathrm{e}^{\mathrm{i} \alpha} w\right) \Longleftrightarrow \Upsilon_{n}(z) \mapsto \mathrm{e}^{\mathrm{i} n \alpha} \Upsilon_{n}(z)
$$

The complex linear superfields $\Gamma^{I}$ can be dualised into chiral superfields ${ }^{10} \Psi_{I}$ which can be interpreted as a one-form at the point $\Phi \in \mathcal{M}[58,15]$. Upon elimination of $\Gamma$ and $\bar{\Gamma}$, the action turns into $S[\Phi, \bar{\Phi}, \Psi, \bar{\Psi}]$. Its target space is (an open neighborhood of the zero section) of the cotangent bundle $T^{*} \mathcal{M}$ of the Kähler manifold $\mathcal{M}$. Since supersymmetry requires this target space to be hyper-Kähler, our consideration is in accord with recent mathematical results [61] about the existence of hyper-Kähler structures on cotangent bundles of Kähler manifolds.

\subsection{Models with intrinsic central charge}

We have so far considered only superconformal multiplets without central charge. As is known, there is no clash between superconformal symmetry and the presence of a central charge provided the latter is gauged. Here we sketch a 5D superspace setting for supersymmetric theories with gauged central charge, which is a natural generalisation of the $4 \mathrm{D} \mathcal{N}=2$ formulation [59].

To start with, one introduces an Abelian vector multiplet, which is destined to gauge the central charge $\Delta$, by defining gauge-covariant derivatives

$$
\mathcal{D}_{\hat{A}}=\left(\mathcal{D}_{\hat{a}}, \mathcal{D}_{\hat{\alpha}}^{i}\right)=D_{\hat{A}}+\mathcal{V}_{\hat{A}}(z) \Delta, \quad\left[\Delta, \mathcal{D}_{\hat{A}}\right]=0
$$

Here the gauge connection $\mathcal{V}_{\hat{A}}$ is inert under the central charge transformations, $\left[\Delta, \mathcal{V}_{\hat{A}}\right]=$ 0 . The gauge-covariant derivatives are required to obey the algebra

$$
\begin{aligned}
& \left\{\mathcal{D}_{\hat{\alpha}}^{i}, \mathcal{D}_{\hat{\beta}}^{j}\right\}=-2 \mathrm{i} \varepsilon^{i j}\left(\mathcal{D}_{\hat{\alpha} \hat{\beta}}+\varepsilon_{\hat{\alpha} \hat{\beta}} \mathcal{W} \Delta\right), \quad\left[\mathcal{D}_{\hat{\alpha}}^{i}, \Delta\right]=0, \\
& {\left[\mathcal{D}_{\hat{\gamma}}^{i}, \mathcal{D}_{\hat{\alpha} \hat{\beta}}\right]=\mathrm{i} \varepsilon_{\hat{\alpha} \hat{\beta}} \mathcal{D}_{\hat{\gamma}}^{i} \mathcal{W} \Delta+2 \mathrm{i}\left(\varepsilon_{\hat{\gamma} \hat{\alpha}} \mathcal{D}_{\hat{\beta}}^{i}-\varepsilon_{\hat{\gamma} \hat{\beta}} \mathcal{D}_{\hat{\alpha}}^{i}\right) \mathcal{W} \Delta,}
\end{aligned}
$$

where the real field strength $\mathcal{W}(z)$ obeys the Bianchi identity (5.19). The field strength should possess a non-vanishing expectation value, $\langle\mathcal{W}\rangle \neq 0$, corresponding to the case of

\footnotetext{
${ }^{10}$ This is accompanied by the appearance of a special chiral superpotential [15].
} 
rigid central charge. By applying a harmonic-dependent gauge transformation, one can choose a frame in which

$$
\mathcal{D}_{\hat{\alpha}}^{+} \rightarrow D_{\hat{\alpha}}^{+}, \quad D^{++} \rightarrow D^{++}+\mathcal{V}^{++} \Delta, \quad D^{--} \rightarrow D^{--}+\mathcal{V}^{--} \Delta
$$

with $\mathcal{V}^{++}$a real analytic prepotential, see [59] for more details. This frame is called the $\lambda$-frame, and the original representation is known as the $\tau$-frame [5].

To generate a supersymmetric action, it is sufficient to construct a real superfield $\mathcal{L}^{(i j)}(z)$ with the properties

$$
\mathcal{D}_{\hat{\alpha}}^{(i} \mathcal{L}^{j k)}=0
$$

which for $\mathcal{L}^{++}(z, u)=\mathcal{L}^{i j}(z) u_{i}^{+} u_{j}^{+}$take the form

$$
\mathcal{D}_{\hat{\alpha}}^{+} \mathcal{L}^{++}=0, \quad D^{++} \mathcal{L}^{++}=0 .
$$

In the $\lambda$-frame, the latter properties become

$$
D_{\hat{\alpha}}^{+} \tilde{\mathcal{L}}^{++}=0, \quad\left(D^{++}+\mathcal{V}^{++} \Delta\right) \tilde{\mathcal{L}}^{++}=0 .
$$

Associated with $\tilde{\mathcal{L}}^{++}$is the supersymmetric action

$$
\int \mathrm{d} \zeta^{(-4)} \mathcal{V}^{++} \tilde{\mathcal{L}}^{++}
$$

which invariant under the central charge gauge transformations $\delta \mathcal{V}^{++}=-D^{++} \lambda$ and $\delta \tilde{\mathcal{L}}^{++}=\lambda \Delta \tilde{\mathcal{L}}^{++}$, with an arbitrary analytic parameter $\lambda$.

Let us give a few examples of off-shell supermultiplets with intrinsic central charge. The simplest is the 5D extension of the Fayet-Sohnius hypermultiplet. It is described by an iso-spinor superfield $\boldsymbol{q}_{i}(z)$ and its conjugate $\overline{\boldsymbol{q}}^{i}(z)$ subject to the constraint

$$
\mathcal{D}_{\hat{\alpha}}^{(i} \boldsymbol{q}^{j)}=0
$$

This multiplet becomes on-shell if $\Delta=$ const. With the notation $\boldsymbol{q}^{+}(z, u)=\boldsymbol{q}^{j}(z) u_{i}^{+}$, the hypermultiplet dynamics is dictated by the Lagrangian

$$
L_{\mathrm{FS}}^{++}=\frac{1}{2} \breve{\boldsymbol{q}}^{+} \overleftrightarrow{\Delta} \boldsymbol{q}^{+}-\mathrm{i} m \breve{\boldsymbol{q}}^{+} \boldsymbol{q}^{+}
$$

with $m$ the hypermultiplet mass/charge. This Lagrangian generates a superconformal theory.

Our second example is an off-shell gauge two-form multiplet called in [3] the massless tensor multiplet. It is Poincaré dual to the $5 \mathrm{D}$ vector multiplet. Similarly to the $4 \mathrm{D}$ 
$\mathcal{N}=2$ vector-tensor multipet [59], it is described by a constrained real superfield $L(z)$ coupled to the central charge vector multiplet. By analogy with the four-dimensional case [59], admissible constraints must obey some nontrivial consistency conditions. In particular, the harmonic-independence of $L$ (in the $\tau$-frame) implies

$$
\begin{aligned}
0=\left(\hat{\mathcal{D}}^{+}\right)^{2}\left(\hat{\mathcal{D}}^{+}\right)^{2} D^{--} L & =D^{--}\left(\hat{\mathcal{D}}^{+}\right)^{2}\left(\hat{\mathcal{D}}^{+}\right)^{2} L-4 \mathcal{D}^{-\hat{\alpha}} \mathcal{D}_{\hat{\alpha}}^{+}\left(\hat{\mathcal{D}}^{+}\right)^{2} L+8 \mathrm{i} \mathcal{D}^{\hat{\alpha} \hat{\beta}} \mathcal{D}_{\hat{\alpha}}^{+} \mathcal{D}_{\hat{\beta}}^{+} \\
& -8 \mathrm{i} \Delta\left\{L\left(\hat{\mathcal{D}}^{+}\right)^{2} \mathcal{W}+\mathcal{W}\left(\hat{\mathcal{D}}^{+}\right)^{2} L+4 \mathcal{D}^{+\hat{\alpha}} \mathcal{W} \mathcal{D}_{\hat{\alpha}}^{+} L\right\}
\end{aligned}
$$

Let us assume that $L$ obeys the constraint

$$
\mathcal{D}_{\hat{\alpha}}^{+} \mathcal{D}_{\hat{\beta}}^{+} L=\frac{1}{4} \varepsilon_{\hat{\alpha} \hat{\beta}}\left(\hat{\mathcal{D}}^{+}\right)^{2} L \quad \Rightarrow \quad \mathcal{D}_{\hat{\alpha}}^{+} \mathcal{D}_{\hat{\beta}}^{+} \mathcal{D}_{\hat{\gamma}}^{+} L=0
$$

which in the case $\Delta=0$ coincides with the Bianchi identity for an Abelian vector multiplet. Then, eq. (6.51) gives

$$
\Delta\left\{L\left(\hat{\mathcal{D}}^{+}\right)^{2} \mathcal{W}+\mathcal{W}\left(\hat{\mathcal{D}}^{+}\right)^{2} L+4 \mathcal{D}^{+\hat{\alpha}} \mathcal{W} \mathcal{D}_{\hat{\alpha}}^{+} L\right\}=0 .
$$

The consistency condition is satisfied if $L$ is constrained as

$$
\left(\hat{\mathcal{D}}^{+}\right)^{2} L=-\frac{1}{\mathcal{W}}\left\{L\left(\hat{\mathcal{D}}^{+}\right)^{2} \mathcal{W}+4 \mathcal{D}^{+\hat{\alpha}} \mathcal{W} \mathcal{D}_{\hat{\alpha}}^{+} L\right\} .
$$

The corresponding Lagrangian is

$$
\mathcal{L}^{++}=-\frac{\mathrm{i}}{4}\left(\mathcal{D}^{+\hat{\alpha}} L \mathcal{D}_{\hat{\alpha}}^{+} L+\frac{1}{2} L\left(\hat{\mathcal{D}}^{+}\right)^{2} L\right) .
$$

The theory generated by this Lagrangian is superconformal.

Another solution to (6.53) describes a Chern-Simons coupling of the two-form multiplet to an external Yang-Mills supermultiplets:

$$
\left(\hat{\mathcal{D}}^{+}\right)^{2} L=-\frac{1}{\mathcal{W}}\left\{L\left(\hat{\mathcal{D}}^{+}\right)^{2} \mathcal{W}+4 \mathcal{D}^{+\hat{\alpha}} \mathcal{W} \mathcal{D}_{\hat{\alpha}}^{+} L\right\}+\frac{\rho}{\mathcal{W}} \mathbb{G}^{++}
$$

where

$$
-\mathrm{i} \mathbb{G}^{++}=\operatorname{tr}\left(\mathcal{D}^{+\hat{\alpha}} \mathbb{W} \mathcal{D}_{\hat{\alpha}}^{+} \mathbb{W}+\frac{1}{4}\left\{\mathbb{W},\left(\hat{\mathcal{D}}^{+}\right)^{2} \mathbb{W}\right\}\right) .
$$

Here $\rho$ is a coupling constant, and $\mathbb{W}$ is the gauge-covariant field strength of the YangMills supermultiplet, see [15] for more details. As the corresponding supersymmetric Lagrangian one can again choose $\mathcal{L}^{++}$given by eq. (6.55). 
A plain dimensional reduction $5 \mathrm{D} \rightarrow 4 \mathrm{D}$ can be shown to reduce the constraints (6.52) and (6.56) to those describing the so-called linear vector-tensor multiplet ${ }^{11}$ with Chern-Simons couplings [59].

When this paper was ready for submission to the hep-th archive, there appeared an interesting work [62] in which 4D and 5D supersymmetric nonlinear sigma models with eight supercharges were formulated in $\mathcal{N}=1$ superspace.

\section{Acknowledgements:}

It is a pleasure to thank Ian McArthur for reading the manuscript. The author is grateful to the Max Planck Institute for Gravitational Physics (Albert Einstein Institute) in Golm and the Institute for Theoretical Physics at the University of Heidelberg for hospitality during the course of the work. This work is supported by the Australian Research Council and by a UWA research grant.

\section{A Non-standard realisation for $S^{2}$}

Let us consider a quantum-mechanical spin-1/2 Hilbert space, i.e. the complex space $\mathbb{C}^{2}$ endowed with the standard positive definite scalar product $\langle\mid\rangle$ defined by

$$
\langle u \mid v\rangle=u^{\dagger} v=\bar{u}^{i} v_{i}, \quad|u\rangle=\left(u_{i}\right)=\left(\begin{array}{c}
u_{1} \\
u_{2}
\end{array}\right), \quad\langle u|=\left(\bar{u}^{i}\right), \quad \bar{u}^{i}=\overline{u_{i}} .
$$

Two-sphere $S^{2}$ can be identified with the space of rays in $\mathbb{C}^{2}$. A ray is represented by a normalized state,

$$
\left|u^{-}\right\rangle=\left(u_{i}^{-}\right), \quad\left\langle u^{-} \mid u^{-}\right\rangle=1, \quad\left\langle u^{-}\right|=\left(u^{+i}\right), \quad u^{+i}=\overline{u_{i}^{-}}
$$

defined modulo the equivalence relation

$$
u_{i}^{-} \sim \mathrm{e}^{-\mathrm{i} \varphi} u_{i}^{-}, \quad\left|\mathrm{e}^{-\mathrm{i} \varphi}\right|=1
$$

Associated with $\left|u^{-}\right\rangle$is another normalized state $\left|u^{+}\right\rangle$,

$$
\left|u^{+}\right\rangle=\left(u_{i}^{+}\right), \quad u_{i}^{+}=\varepsilon_{i j} u^{+j}, \quad\left\langle u^{+} \mid u^{+}\right\rangle=1
$$

\footnotetext{
${ }^{11}$ Ref. [59] contains an extensive list of publications on the linear and nonlinear vector-tensor multiplets and their couplings.
} 
which is orthogonal to $\left|u^{-}\right\rangle$,

$$
\left\langle u^{+} \mid u^{-}\right\rangle=0
$$

The states $\left|u^{-}\right\rangle$and $\left|u^{+}\right\rangle$generate the unimodular unitary matrix

$$
\boldsymbol{u}=\left(\left|u^{-}\right\rangle,\left|u^{+}\right\rangle\right)=\left(u_{i}^{-}, u_{i}^{+}\right) \in S U(2) .
$$

In terms of this matrix, the equivalence relation (A.3) becomes

$$
\boldsymbol{u} \sim \boldsymbol{u}\left(\begin{array}{cc}
\mathrm{e}^{-\mathrm{i} \varphi} & 0 \\
0 & \mathrm{e}^{\mathrm{i} \varphi}
\end{array}\right)
$$

This gives the well-known realisation $S^{2}=S U(2) / U(1)$.

The above unitary realisation for $S^{2}$ is ideal if one is interested in the action of $S U(2)$, or its subgroups, on the two-sphere. But it is hardly convenient if one considers, for instance, the action of $S L(2, \mathbb{C})$ on $S^{2}$. There exists, however, a universal realisation. Instead of dealing with the orthonormal basis $\left(\left|u^{-}\right\rangle,\left|u^{+}\right\rangle\right)$introduced, one can work with an arbitrary basis for $\mathbb{C}^{2}$ :

$$
\boldsymbol{v}=\left(\left|v^{-}\right\rangle,\left|v^{+}\right\rangle\right)=\left(v_{i}^{-}, v_{i}^{+}\right) \in G L(2, \mathbb{C}), \quad \operatorname{det} \boldsymbol{v}=v^{+i} v_{i}^{-}
$$

The two-sphere is then obtained by factorisation with respect to the equivalence relation

$$
\boldsymbol{v} \sim \boldsymbol{v} R, \quad R=\left(\begin{array}{ll}
a & 0 \\
b & c
\end{array}\right) \in G L(2, \mathbb{C}) .
$$

Given an arbitrary matrix $\boldsymbol{v} \in G L(2, \mathbb{C})$, there always exists a lower triangular matrix $R$ such that $\boldsymbol{v} R \in S U(2)$, and then we are back to the unitary realisation. One can also consider an intermediate realisation for $S^{2}$ given in terms of unimodular matrices of the form

$$
\boldsymbol{w}=\left(\left|w^{-}\right\rangle,\left|w^{+}\right\rangle\right)=\left(w_{i}^{-}, w_{i}^{+}\right) \in S L(2, \mathbb{C}) \quad \longleftrightarrow \quad w^{+i} w_{i}^{-}=1
$$

and the matrix $R$ in (A.9) should be restricted to be unimodular. The harmonics $w^{ \pm}$are complex in the sense that $w_{i}^{-}$and $w^{+i}$ are not related by complex conjugation.

Let us consider a left group transformation acting on $S^{2}$

$$
\boldsymbol{w} \rightarrow g \boldsymbol{w}=\left(v_{i}^{-}, v_{i}^{+}\right) \equiv \boldsymbol{v}
$$

If $g$ is a "small" transformation, i.e. if it belongs to a sufficiently small neighbourhood of the identity, then there exists a matrix $R$ of the type (A.9) such that

$$
g \boldsymbol{w} R=\left(w_{i}^{-}, \hat{w}_{i}^{+}\right) \in S L(2, \mathbb{C}) .
$$


Since

$$
w^{+i} w_{i}^{-}=1, \quad \hat{w}^{+i} w_{i}^{-}=1,
$$

for the transformed harmonic we thus obtain

$$
\hat{w}_{i}^{+}=w_{i}^{+}+\rho^{++}(w) w_{i}^{-} .
$$

All information about the group transformation $g$ is now encoded in $\rho^{++}$.

\section{B Projective superspace action}

Following [8], consider

$$
I=\frac{1}{2 \pi \mathrm{i}} \oint \frac{u_{i}^{+} \mathrm{d} u^{+i}}{\left(u^{+} u^{-}\right)^{4}} \int \mathrm{d}^{5} x\left(\hat{D}^{-}\right)^{4} \mathcal{L}^{++}\left(z, u^{+}\right),
$$

where the Lagrangian enjoys the properties

$$
D_{\hat{\alpha}}^{+} \mathcal{L}^{++}\left(z, u^{+}\right)=0, \quad \mathcal{L}^{++}\left(z, c u^{+}\right)=c^{2} \mathcal{L}^{++}\left(z, u^{+}\right), \quad c \in \mathbb{C}^{*} .
$$

The functional (B.1) is invariant under arbitrary projective transformations (4.10). Choosing the projective gauge (4.23) gives the supersymmetric action (6.19).

It is worth pointing out that the vector multiplet field strength (5.38) can be rewritten in the projective-invariant form

$$
W(z)=-\frac{1}{16 \pi \mathrm{i}} \oint \frac{u_{i}^{+} \mathrm{d} u^{+i}}{\left(u^{+} u^{-}\right)^{2}}\left(\hat{D}^{-}\right)^{2} V\left(z, u^{+}\right),
$$

where the gauge potential enjoys the properties

$$
D_{\hat{\alpha}}^{+} V\left(z, u^{+}\right)=0, \quad V\left(z, c u^{+}\right)=V\left(z, u^{+}\right), \quad c \in \mathbb{C}^{*} .
$$

\section{From 5D projective supermultiplets to $4 \mathrm{D} \mathcal{N}=1,2$ superfields}

The conventional 5D simple superspace $\mathbb{R}^{5 \mid 8}$ is parametrized by coordinates $z^{\hat{A}}=$ $\left(x^{\hat{a}}, \theta_{i}^{\hat{\alpha}}\right)$, with $i=\underline{1}, \underline{2}$. Any hypersurface $x^{5}=$ const in $\mathbb{R}^{5 / 8}$ can be identified with the 
$4 \mathrm{D}, \mathcal{N}=2$ superspace $\mathbb{R}^{4 \mid 8}$ parametrized by $z^{A}=\left(x^{a}, \theta_{i}^{\alpha}, \bar{\theta}_{\dot{\alpha}}^{i}\right)$, where $\left(\theta_{i}^{\alpha}\right)^{*}=\bar{\theta}^{\dot{\alpha} i}$. The Grassmann coordinates of $\mathbb{R}^{5 \mid 8}$ and $\mathbb{R}^{4 \mid 8}$ are related to each other as follows:

$$
\theta_{i}^{\hat{\alpha}}=\left(\theta_{i}^{\alpha},-\bar{\theta}_{\dot{\alpha} i}\right), \quad \theta_{\hat{\alpha}}^{i}=\left(\begin{array}{c}
\theta_{\alpha}^{i} \\
\bar{\theta}^{\dot{\alpha} i}
\end{array}\right) .
$$

Interpreting $x^{5}$ as a central charge variable, one can view $\mathbb{R}^{5 \mid 8}$ as a $4 \mathrm{D}, \mathcal{N}=2$ central charge superspace. One can relate the 5D spinor covariant derivatives (see [15] for more details)

$$
D_{\hat{\alpha}}^{i}=\left(\begin{array}{c}
D_{\alpha}^{i} \\
\bar{D}^{\dot{\alpha} i}
\end{array}\right)=D_{\hat{\alpha}}^{i}=\frac{\partial}{\partial \theta_{i}^{\hat{\alpha}}}-\mathrm{i}\left(\Gamma^{\hat{b}}\right)_{\hat{\alpha} \hat{\beta}} \theta^{\hat{\beta} i} \partial_{\hat{b}}, \quad D_{i}^{\hat{\alpha}}=\left(D_{i}^{\alpha},-\bar{D}_{\dot{\alpha} i}\right)
$$

to the $4 \mathrm{D}, \mathcal{N}=2$ covariant derivatives $D_{A}=\left(\partial_{a}, D_{\alpha}^{i}, \bar{D}_{i}^{\dot{\alpha}}\right)$ where

$$
D_{\alpha}^{i}=\frac{\partial}{\partial \theta_{i}^{\alpha}}+\mathrm{i}\left(\sigma^{b}\right)_{\alpha \dot{\beta}} \bar{\theta}^{\dot{\beta} i} \partial_{b}+\theta_{\alpha}^{i} \partial_{5}, \quad \bar{D}_{\dot{\alpha} i}=-\frac{\partial}{\partial \bar{\theta}^{\dot{\alpha} i}}-\mathrm{i} \theta_{i}^{\beta}\left(\sigma^{b}\right)_{\beta \dot{\alpha}} \partial_{b}-\bar{\theta}_{\dot{\alpha} i} \partial_{5}
$$

These operators obey the anti-commutation relations

$$
\begin{gathered}
\left\{D_{\alpha}^{i}, D_{\beta}^{j}\right\}=2 \varepsilon^{i j} \varepsilon_{\alpha \beta} \partial_{5}, \quad\left\{\bar{D}_{\dot{\alpha} i}, \bar{D}_{\dot{\beta} j}\right\}=2 \varepsilon_{i j} \varepsilon_{\dot{\alpha} \dot{\beta}} \partial_{5}, \\
\left\{D_{\alpha}^{i}, \bar{D}_{\dot{\beta} j}\right\}=-2 \mathrm{i} \delta_{j}^{i}\left(\sigma^{c}\right)_{\alpha \dot{\beta}} \partial_{c},
\end{gathered}
$$

which correspond to the $4 \mathrm{D}, \mathcal{N}=2$ supersymmetry algebra with the central charge $\partial_{5}$.

Consider a 5D projective superfield (5.33). Representing the differential operators $\nabla_{\hat{\alpha}}(w)$, eq. $(5.32)$, as

$$
\nabla_{\hat{\alpha}}(w)=\left(\begin{array}{c}
\nabla_{\alpha}(w) \\
\bar{\nabla}^{\dot{\alpha}}(w)
\end{array}\right), \quad \nabla_{\alpha}(w) \equiv w D_{\alpha}^{\underline{1}}-D_{\alpha}^{\underline{\alpha}}, \quad \bar{\nabla}^{\dot{\alpha}}(w) \equiv \bar{D}_{\underline{1}}^{\dot{\alpha}}+w \bar{D}_{\underline{2}}^{\dot{\alpha}},
$$

the constraints (5.34) can be rewritten in the component form

$$
D_{\alpha}^{2} \phi_{n}=D_{\alpha}^{\frac{1}{\alpha}} \phi_{n-1}, \quad \bar{D}_{\underline{2}}^{\dot{\alpha}} \phi_{n}=-\bar{D}_{\underline{1}}^{\dot{\alpha}} \phi_{n+1} .
$$

The relations (C.6) imply that the dependence of the component superfields $\phi_{n}$ on $\theta_{\underline{2}}^{\alpha}$ and $\bar{\theta}_{\dot{\alpha}}^{2}$ is uniquely determined in terms of their dependence on $\theta_{\underline{1}}^{\alpha}$ and $\bar{\theta} \frac{1}{\dot{\alpha}}$. In other words, the projective superfields depend effectively on half the Grassmann variables which can be choosen to be the spinor coordinates of $4 \mathrm{D} \mathcal{N}=1$ superspace

$$
\theta^{\alpha}=\theta_{\underline{1}}^{\alpha}, \quad \bar{\theta}_{\dot{\alpha}}=\bar{\theta} \frac{1}{\dot{\alpha}} .
$$

Then, one deals with reduced superfields $\phi\left|, D_{\alpha}^{2} \phi\right|, \bar{D}_{\underline{2}}^{\dot{\alpha}} \phi \mid, \ldots$ (of which not all are usually independent) and $4 \mathrm{D} \mathcal{N}=1$ spinor covariant derivatives $D_{\alpha}$ and $\bar{D}^{\dot{\alpha}}$ defined in the obvious way:

$$
\phi\left|=\phi\left(x, \theta_{i}^{\alpha}, \bar{\theta}_{\dot{\alpha}}^{i}\right)\right|_{\theta_{\underline{2}}=\bar{\theta} \underline{2}=0}, \quad D_{\alpha}=\left.D \frac{1}{\alpha}\right|_{\theta_{\underline{2}}=\bar{\theta} \underline{2}=0}, \quad \bar{D}^{\dot{\alpha}}=\left.\bar{D}_{\underline{1}}^{\dot{\alpha}}\right|_{\theta_{\underline{2}}=\bar{\theta} \underline{2}=0} .
$$




\section{References}

[1] W. Nahm, "Supersymmetries and their representations," Nucl. Phys. B 135 (1978) 149.

[2] V. G. Kac, "Lie superalgebras," Adv. Math. 26 (1977) 8.

[3] T. Kugo and K. Ohashi, "Off-shell d $=5$ supergravity coupled to matter-Yang-Mills system," Prog. Theor. Phys. 105 (2001) 323 [hep-ph/0010288]; T. Fujita and K. Ohashi, "Superconformal tensor calculus in five dimensions," Prog. Theor. Phys. 106 (2001) 221 [hep-th/0104130]; T. Fujita, T. Kugo and K. Ohashi, "Off-shell formulation of supergravity on orbifold," Prog. Theor. Phys. 106 (2001) 671 [hep-th/0106051]; T. Kugo and K. Ohashi, "Superconformal tensor calculus on orbifold in 5D," Prog. Theor. Phys. 108 (2002) 203 [arXiv:hep-th/0203276].

[4] E. Bergshoeff, S. Cucu, T. De Wit, J. Gheerardyn, R. Halbersma, S. Vandoren and A. Van Proeyen, "Superconformal N $=2, \mathrm{D}=5$ matter with and without actions," JHEP 0210 (2002) 045 [hep-th/0205230]; E. Bergshoeff, S. Cucu, T. de Wit, J. Gheerardyn, S. Vandoren and A. Van Proeyen, "N = 2 supergravity in five dimensions revisited," Class. Quant. Grav. 21 (2004) 3015 [arXiv:hep-th/0403045].

[5] A. Galperin, E. Ivanov, S. Kalitsyn, V. Ogievetsky and E. Sokatchev, "Unconstrained N = 2 matter, Yang-Mills and supergravity theories in harmonic superspace," Class. Quant. Grav. 1 (1984) 469.

[6] A. S. Galperin, E. A. Ivanov, V. I. Ogievetsky and E. S. Sokatchev, Harmonic Superspace, Cambridge University Press, Cambridge, 2001.

[7] A. Karlhede, U. Lindström and M. Roček, "Self-interacting tensor multiplets in N = 2 superspace," Phys. Lett. B 147 (1984) 297.

[8] W. Siegel, "Chiral actions for $\mathrm{N}=2$ supersymmetric tensor multiplets," Phys. Lett. B 153 (1985) 51.

[9] U. Lindström and M. Roček, "New hyperkahler metrics and new supermultiplets," Commun. Math. Phys. 115 (1988) 21; "N = 2 super Yang-Mills theory in projective superspace," Commun. Math. Phys. 128 (1990) 191; F. Gonzalez-Rey, M. Roček, S. Wiles, U. Lindström and R. von Unge, "Feynman rules in $\mathrm{N}=2$ projective superspace. I: Massless hypermultiplets," Nucl. Phys. B 516 (1998) 426 [hep-th/9710250].

[10] N. Berkovits and W. Siegel, "Superspace effective actions for 4D compactifications of heterotic and type II superstrings," Nucl. Phys. B 462 (1996) 213 [hep-th/9510106]; N. Berkovits, "Conformal compensators and manifest type IIB S-duality," Phys. Lett. B 423 (1998) 265 [hep-th/9801009]. 
[11] A. A. Rosly, "Super Yang-Mills constraints as integrability conditions," in Group Theoretical Methods in Physics," M. A. Markov (Ed.), Nauka, Moscow, 1983, p. 263.

[12] A. A. Rosly and A. S. Schwarz, "Supersymmetry in a space with auxiliary dimensions," Commun. Math. Phys. 105, 645 (1986).

[13] E. Witten, "An interpretation of classical Yang-Mills theory," Phys. Lett. B 77 (1978) 394.

[14] S. M. Kuzenko, "Projective superspace as a double-punctured harmonic superspace," Int. J. Mod. Phys. A 14 (1999) 1737 [hep-th/9806147].

[15] S. M. Kuzenko and W. D. Linch, "On five-dimensional superspaces," hep-th/0507176.

[16] B. de Wit, M. Roček and S. Vandoren, "Hypermultiplets, hyperkaehler cones and quaternion-Kaehler geometry," JHEP 0102 (2001) 039 [hep-th/0101161].

[17] A. S. Galperin, E. A. Ivanov, V. I. Ogievetsky and E. S. Sokatchev, "Conformal invariance in harmonic superspace," in Quantum Field Theory and Quantum Statistics, I. Batalin, C. J. Isham and G. Vilkovisky (Eds.), Vol. 2, Adam Hilger, Bristol, 1987.

[18] E. A. Ivanov, A. V. Smilga and B. M. Zupnik, "Renormalizable supersymmetric gauge theory in six dimensions," Nucl. Phys. B 726 (2005) 131 [hep-th/0505082].

[19] A. A. Rosly, "Gauge fields in superspace and twistors," Class. Quant. Grav. 2 (1985) 693.

[20] J. Lukierski and A. Nowicki, "General superspaces from supertwistors," Phys. Lett. B 211 (1988) 276.

[21] P. S. Howe and G. G. Hartwell, "A superspace survey," Class. Quant. Grav. 12 (1995) 1823.

[22] Yu. I. Manin, Gauge Field Theory and Complex Geometry, Springer, Berlin, 1988.

[23] R. Penrose, "Twistor algebra," J. Math. Phys. 8 (1967) 345; R. Penrose and M. A. H. MacCallum, "Twistor theory: An approach to the quantization of fields and space-time," Phys. Rept. 6 (1972) 241.

[24] R. S. Ward and R. O. Wells, Twistor Geometry and Field Theory, Cambridge University Press, Cambridge, 1991.

[25] G. W. Gibbons and A. R. Steif, "Sphalerons and conformally compactified Minkowski space-time," Phys. Lett. B 346 (1995) 255 [hep-ph/9412210].

[26] I. E. Segal, Mathematical Cosmology and Extragalactic Astronomy, Academic Press, New York, 1976. 
[27] O. Aharony, S. S. Gubser, J. M. Maldacena, H. Ooguri and Y. Oz, "Large N field theories, string theory and gravity," Phys. Rept. 323 (2000) 183 [hep-th/9905111].

[28] M. F. Sohnius, "The conformal group in superspace," in Quantum Theory and the Structures of Time and Space, Vol. 2, L. Castell, M. Drieschner and C. F. von Weizsäcker (Eds.), Carl Hanser Verlag, München, 1977, p. 241.

[29] W. Lang, "Construction of the minimal superspace translation tensor and the derivation of the supercurrent," Nucl. Phys. B 179 (1981) 106.

[30] L. Bonora, P. Pasti and M. Tonin, "Cohomologies and anomalies in supersymmetric theories," Nucl. Phys. B 252 (1985) 458.

[31] K. i. Shizuya, "Supercurrents and superconformal symmetry," Phys. Rev. D 35 (1987) 1848.

[32] I. L. Buchbinder and S. M. Kuzenko, Ideas and Methods of Supersymmetry and Supergravity or a Walk Through Superspace, IOP, Bristol, 1998.

[33] P. C. West, "Introduction to rigid supersymmetric theories," hep-th/9805055.

[34] H. Osborn, "N = 1 superconformal symmetry in four-dimensional quantum field theory," Annals Phys. 272 (1999) 243 [hep-th/9808041].

[35] J. H. Park, "Superconformal symmetry in six-dimensions and its reduction to four," Nucl. Phys. B 539 (1999) 599 [hep-th/9807186]; "Superconformal symmetry and correlation functions," Nucl. Phys. B 559 (1999) 455 [hep-th/9903230].

[36] S. M. Kuzenko and S. Theisen, "Correlation functions of conserved currents in $\mathrm{N}=2$ superconformal theory," Class. Quant. Grav. 17 (2000) 665 [hep-th/9907107].

[37] A. Uhlmann, "The closure of Minkowski space," Acta Phys. Pol. 24 (1963) 295.

[38] I. T. Todorov, M. C. Mintchev and V. P. Petkova, Conformal Invariance in Quantum Field Theory, Pisa, Scuola Normale Superiore, 1978; I. T. Todorov, Conformal Description of Spinning Particles, Springer, Berlin, 1986.

[39] H. Weyl, Space-Time-Matter, Dover Publications, New York, 1952.

[40] P. A. M. Dirac, "Wave equations in conformal space," Ann. Math. 37 (1936) 429.

[41] É. Cartan, "Sur les domaines bornés homogènes de l'espace de $n$ variables complexes," Abh. Math. Sem. Univ. Hamburg 11 (1935) 116.

[42] L. K. Hua, Harmonic Analysis of Functions of Several Complex Variables in the Classical Domains, American Mathematical Society, Providence, 1963. 
[43] S. M. Paneitz and I. E. Segal, "Analysis in space-time bundles. I. General considerations and the scalar bundle," J. Funct. Anal. 47 (1982) 78.

[44] J. Wess and J. Bagger, Supersymmetry and Supergravity, Princeton Univ. Press, 1992.

[45] A. Ferber, "Supertwistors and conformal supersymmetry," Nucl. Phys. B 132 (1978) 55.

[46] B. S. DeWitt, Supermanifolds, Cambridge University Press, Cambridge, 1992.

[47] B. Zupnik, "Harmonic superpotentials and symmetries in gauge theories with eight supercharges," Nucl. Phys. B 554 (1999) 365 [hep-th/9902038].

[48] P. S. Howe, G. Sierra and P. K. Townsend, "Supersymmetry in six dimensions," Nucl. Phys. B 221 (1983) 331.

[49] P. S. Howe, K. S. Stelle and P. C. West, "N=1 D =6 harmonic superspace," Class. Quant. Grav. 2 (1985) 815; B. M. Zupnik, Sov. J. Nucl. Phys. 44 (1986) 512.

[50] J. Grundberg and U. Lindstrom, "Actions for linear multiplets in six dimensions," Class. Quant. Grav. 2 (1985) L33; S. J. Gates, S. Penati and G. Tartaglino-Mazzucchelli, "6D supersymmetry, projective superspace and 4D, $\mathrm{N}=1$ superfields," hep-th/0508187.

[51] B. de Wit, R. Philippe and A. Van Proeyen, "The improved tensor multiplet in N = 2 supergravity," Nucl. Phys. B 219 (1983) 143.

[52] U. Lindstrom and M. Roček, "Scalar tensor duality and N = 1, 2 nonlinear sigma models," Nucl. Phys. B 222 (1983) 285.

[53] A. Galperin, E. Ivanov and V. Ogievetsky, "Superspace actions and duality transformations for N = 2 tensor multiplets," Sov. J. Nucl. Phys. 45 (1987) 157; "Duality transformations and most general matter self-coupling in N = 2 supersymmetry," Nucl. Phys. B 282 (1987) 74.

[54] S. M. Kuzenko, "On massive tensor multiplets," JHEP 0501 (2005) 041 [hep-th/0412190].

[55] B. de Wit, M. T. Grisaru and M. Roček, "Nonholomorphic corrections to the one-loop N=2 super Yang-Mills action,” Phys. Lett. B 374 (1996) 297 [hep-th/9601115].

[56] I. L. Buchbinder, S. M. Kuzenko and A. A. Tseytlin, "On low-energy effective actions in $\mathrm{N}=2,4$ superconformal theories in four dimensions," Phys. Rev. D 62 (2000) 045001 [hep-th/9911221].

[57] M. Henningson, "Extended superspace, higher derivatives and SL(2,Z) duality," Nucl. Phys. B 458 (1996) 445 [hep-th/9507135]. 
[58] S. J. Gates and S. M. Kuzenko, "The CNM-hypermultiplet nexus," Nucl. Phys. B 543 (1999) 122 [hep-th/9810137]; "4D N = 2 supersymmetric off-shell sigma models on the cotangent bundles of Kähler manifolds," Fortsch. Phys. 48 (2000) 115 [hep-th/9903013].

[59] N. Dragon, E. Ivanov, S. Kuzenko, E. Sokatchev and U. Theis, "N = 2 rigid supersymmetry with gauged central charge," Nucl. Phys. B 538 (1999) 411 [hep-th/9805152].

[60] B. de Wit, B. Kleijn and S. Vandoren, "Rigid N = 2 superconformal hypermultiplets," in Supersymmetries and Quantum Symmetries, J. Wess and E. A. Ivanov (Eds.), Springer, Berlin, 1999, p. 37, hep-th/9808160; "Superconformal hypermultiplets," Nucl. Phys. B 568 (2000) 475 [hep-th/9909228].

[61] D. Kaledin, "Hyperkähler structures on total spaces of holomorphic cotangent bundles," in D. Kaledin and M. Verbitsky, Hyperkähler Manifolds, International Press, Cambridge MA, 1999 [alg-geom/9710026]; B. Feix, "Hyperkähler metrics on cotangent bundles," Cambridge PhD thesis, 1999; J. reine angew. Math. 532, 33 (2001).

[62] J. Bagger and C. Xiong, " $\mathrm{N}=2$ nonlinear sigma models in $\mathrm{N}=1$ superspace: Four and five dimensions," hep-th/0601165. 